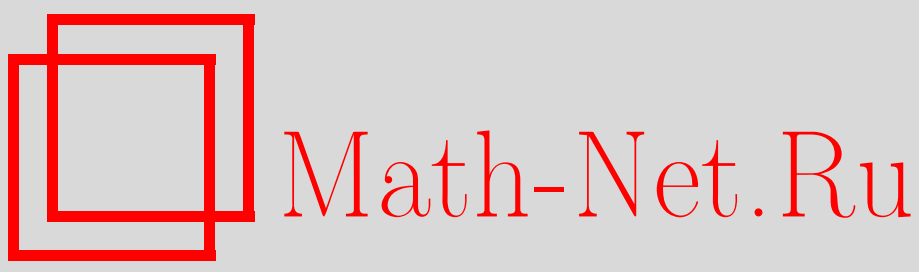

А. В. Романов, Конечномерность динамики на аттракторе для нелинейных параболических уравнений, Изв. РАН. Сер. матем., 2001, том 65, выпуск 5, 129-152

DOI: https://doi.org/10.4213/im359

Использование Общероссийского математического портала Math-Net.Ru подразумевает, что вы прочитали и согласны с пользовательским соглашением

http://www . mathnet.ru/rus/agreement

Параметры загрузки:

IP : 54.198 .187 .58

26 апреля 2023 г., 07:54:39 
УДК 517.95

\author{
А. В. Романов
}

\title{
Конечномерность динамики на аттракторе для нелинейных параболических уравнений
}

\begin{abstract}
Показано, что одномерные полулинейные параболические уравнения второго порядка обладают свойством конечномерности динамики на аттракторе. В том числе, это верно для уравнений реакции-диффузии с конвекцией на $(0,1)$.

Найдены новые топологические критерии конечномерности динамики на инвариантных компактах для класса диссипативных уравнений параболического типа в пространствах Банаха. Динамика таких уравнений на аттракторе $\mathscr{A}$ конечномерна (описывается некоторым ОДУ), если $\mathscr{A}$ можно вложить в конечномерное $C^{1}$-подмногообразие фазового пространства.
\end{abstract}

Библиографиял: 29 наименований.

\section{Введение}

Настояшая статья, как и предшествуюшая ей работа [1], посвящена одному новому подходу к изучению предельных режимов полулинейных параболических уравнений

$$
\partial_{t} u=-A u+F(u), \quad u=u(t),
$$

в банаховом пространстве $X$ с линейным неограниченным секториальным оператором $A$ и "относительно слабой" нелинейностью $F$. Предполагаем, что (1) порождает гладкий диссипативный полупоток в $X^{\alpha}$, где $0 \leqslant \alpha<1$, a $\left\{X^{\theta}\right\}_{\theta \in \mathbb{R}}-$ определяемая оператором $A$ шкала пространств Банаха [2].

Обсуждаются условия, при которых динамика (1) на инвариантном компакте $\mathscr{K} \subset X^{\alpha}$ может быть описана обыкновенным дифференциальным уравнением (ОДУ) с липшицевым векторным полем в $\mathbb{R}^{n}$, что подразумевает липшиц-сопряженность соответствуюших фазовых полупотоков на $\mathscr{K}$ и некотором инвариантном для ОДУ компакте $\mathscr{K}_{1} \subset \mathbb{R}^{n}$. В этом случае говорим о конечномерной динамике на $\mathscr{K}$. Если уравнение (1) обладает компактным аттрактором $\mathscr{A} \subset$ $X^{\alpha}$ - инвариантным множеством, равномерно притягивающим шары $\mathscr{B} \subset X^{\alpha}$ при $t \rightarrow+\infty$, то наибольший интерес представляет эффект конечномерности динамики на $\mathscr{A}$, или, в терминологии [1], конечномерности предельной динамики (1).

Гипотезу о конечномерном характере финальных режимов нелинейных параболических уравнений высказьвал уже Хопф [3]. Первые конкретные результаты в этом направлении (типа "конечности числа определяющих мод") получили Фойяш, Проди [4] и Ладыженская [5], причем в работе [5] было установлено наличие компактного аттрактора $\mathscr{A}$ для широкого класса задач (1). Конечномерность динамики на $\mathscr{A}$ трактовалась тогда О. А. Ладыженской как возможность восстановления полных траекторий $\{u(t)\}_{t \in \mathbb{R}} \subset \mathscr{A}$ по их проекции в подходящее конечномерное подпространство $Y \subset X^{\alpha}$. Удобным инструментом доказательства конеч-

(C) А. В. РомАнов, 2001 
номерности инвариантных компактов (1) стала общая теорема Малле-Паре [6] о гладких вполне непрерывных отображениях в гильбертовом пространстве, а также ее банахова версия [7]. Различные методы оценки размерности (хаусдорфовой, фрактальной, ляпуновской) аттракторов эволюционных уравнений изложены в [8]-[10]. Цельный взгляд на проблему конечнопараметрического отслеживания траекторий распределенных динамических систем представлен не так давно Чуешовым [11].

В работе Мане [12] для уравнений (1) в гильбертовом пространстве с самосопряженным оператором $A$ было сформулировано (см. также [10], [13]) так назьваемое условие спектрального скачка, позволяющее построить инерциальное многообразие $\mathscr{M} \subset X^{\alpha}$. Речь идет о гладкой или липшицевой конечномерной инвариантной поверхности, содержащей аттрактор $\mathscr{A}$ и притягивающей шары $\mathscr{B} \subset X^{\alpha}$ с экспоненциальной скоростью. При этом $\mathscr{M}$ устроено как график, и сужение уравнения (1) на $\mathscr{M}$ дает инерциальную форму - ОДУ в $\mathbb{R}^{n}$, моделирующее не только точное поведение решений $u(t)$ на $\mathscr{A}$, но и (асимптотически по $t \rightarrow+\infty$ ) фазовую динамику (1) в $X^{\alpha}$. Как видим, существование инерциального многообразия ("асимптотическая конечномерность") является несколько более сильным свойством, чем конечномерность предельной динамики. К сожалению, условие спектрального скачка, предполагающее значительную разреженность спектра $A$, оказалось весьма ограничительным, и к настоящему моменту теория инерциальных многообразий зашла, по существу, в тупик.

В то же время есть основания полагать, что для конкретной диссипативной системы доказать конечномерность динамики на аттракторе иногда проще, чем установить наличие инерциального многообразия. В качестве первого (и, конечно, не последнего) подтверждающего примера здесь предлагаются одномерные параболические уравнения

$$
u_{t}=u_{x x}+f\left(x, u, u_{x}\right), \quad x \in(0,1),
$$

при разделенных или периодических краевых условиях с гладкой функцией $f$, чьи характеристики гарантируют глобальное (по $t>0)$ существование и надлежащие оценки решений смешанной задачи. Самосопряженный полуограниченный снизу оператор $A=-\partial_{x x}$ в $X=L^{2}(0,1)$ определяет гильбертову шкалу пространств $\left\{X^{\theta}\right\}$. Считаем, что при некотором $\alpha \in(3 / 4,1)$ уравнение $(2)$ порождает в $X^{\alpha}$ гладкий полупоток и обладает компактным аттрактором $\mathscr{A} \subset X^{\alpha}$. Один из главных выводов статьи (теорема 3.3) состоит в том, что фазовая динамика (2) на $\mathscr{A}$ конечномерна. Между тем, до сих пор неизвестно, всегда ли существует в данной ситуации инерциальное многообразие. Согласно [1] конечномерность предельной динамики [2] имеет такие следствия: векторное поле уравнения лишшицево на $\mathscr{A}$ в $X^{\alpha}$-метрике, а полупоток расширяется на $\mathscr{A}$ до липшицева в $X^{\alpha}$-метрике потока; аттрактор $\mathscr{A}$ есть часть конечномерного липшищева многообразия (типа графика) $\mathscr{M} \subset X^{\alpha}$. С другой стороны, уравнение (2) на окружности допускает периодические по $t$ решения $u(t, \cdot)$ и конечномерность динамики на аттракторе позволяет утверждать, что периоды таких решений ограничены снизу положительной постоянной $c=c(f)$.

Конечномерность предельной динамики можно получить также для систем уравнений вида $(2)$ с $u(0)=u(1)=0$ и с $\left(d(x) u_{x}\right)_{x}$ вместо $u_{x x}$, где $d(x)>0$ на 
$[0,1]$ - гладкий, единый по всем компонентам системы, неоднородный "коэффиициент диффузии".

Базой доказательства теоремы 3.3 служат результаты $\S 1,2$ для абстрактного уравнения (1), представляюшие самостоятельный интерес. Пусть $A$ - дискретньй секториальный оператор в $X$, функция $F$ принадлежит $C^{2}\left(X^{\alpha}, X\right)$ при каком-то $\alpha \in[0,1)$ и ограничена на шарах $X^{\alpha},|\cdot|_{\alpha}$ - норма в $X^{\alpha}$. Tеорема 1.4 устанавливает два новых, по сравнению с [1], критерия конечномерности динамики (1) на инвариантных компактах $\mathscr{K} \subset X^{\alpha}$. Первьй из них - относительная компактность в $X^{\alpha}$ множества точек $w=(u-v) /|u-v|_{\alpha}, u, v \in \mathscr{K}, u \neq v$. Другой сводится к наличию для каждой точки $w \in \mathscr{K}$ такой $X^{\alpha}$-окрестности $\mathscr{V} \supset w$ и такого конечномерного непрерывного в $X^{\alpha}$ проектора $P$, что

$$
|u-v|_{\alpha} \leqslant c|P(u-v)|_{\alpha}
$$

на $\mathscr{V} \cap \mathscr{K}, c=c(\mathscr{K}, w, P)$. Если пространство $X$ нерефлексивно, то нужна еше непрерывность $P$ в $X^{\alpha-1}$. Далее, теорема 1.5 описывает связь между конечномерностью динамики на инвариантном компакте $\mathscr{K} \subset X^{\alpha}$ и вложимостью (тождественной) $\mathscr{K}$ в достаточно регулярное конечномерное подмногообразие $\mathscr{M} \subset X^{\alpha}$. Именно, $C^{1}$-гладкость $\mathscr{M}$ обеспечивает конечномерность фазовой динамики на $\mathscr{K}$; обратное же верно, если $\mathscr{M}$ - липшицево многообразие. Подчеркнем, что многообразие $\mathscr{M}$ не предполагается инвариантным.

Заметим, что для справедливости некоторых из перечисленных утверждений дополнительно требуется базисность в $X^{\alpha}$ конечномерных инвариантных подпространств оператора $A$, упорядоченных определенным образом.

Все эти построения носят топологический характер, но в гильбертовом случае теоремы $2.3,2.8$ дают и аналитические условия на векторное поле $G(u)=$ $F(u)-A u$ уравнения (1), гарантирующие конечномерность его предельной динамики. Указанные условия подразумевают декомпозицию

$$
G(u)-G(v)=\left(B_{0}(u, v)-B(u, v)\right)(u-v)
$$

на аттракторе $\mathscr{A} \subset X^{\alpha}$, где $B_{0}(u, v)$ - поле линейных непрерывных отображений $X^{\alpha} \rightarrow X^{\alpha}$, а $B(u, v)$ - поле линейных неограниченных секториальных операторов в $X$, подобных нормальным. Требуется также достаточная (но при $\alpha \neq 0$ меньшая, чем для спектра $\sigma(A)$ в условии спектрального скачка) разреженность множества $\Sigma=\bigcup_{u, v \in \mathscr{A}} \sigma(B(u, v))$. Отсюда при некоторых технических ограничениях на поля операторов $B_{0}, B$ делается вывод о применимости второго критерия теоремы 1.4 и, стало быть, о конечномерности динамики на $\mathscr{A}$.

Такой сценарий успешно реализуется для одномерных полулинейных параболических уравнений (2), однако соответствующий переход к размерности $\geqslant 2$ остается проблемным даже в “простой" ситуации $f=f(x, u)$. Не удается пока установить и конечномерность предельной динамики для двумерной системы Навье-Стокса. Между тем, аргументация работы $[1 ; \S 4]$ позволяет рассчитьвать, в принципе, на положительное решение данной проблемы при периодических краевых условиях. Дальнейшие усилия должны быть направлены на ослабление довольно жестких предположений теорем 2.3 и 2.8, что позволит расширить список уравнений параболического типа, демонстрирующих конечномерную динамику на аттракторе. 
Естественно возникающий вопрос о существовании уравнений (1), не обладаюших конечномерной предельной динамикой, вряд ли решается просто. По крайней мере в сходной ситуации с асимптотической конечномерностью фазовой динамики ответ (утвердительньй) на вопрос подобного рода получен лиш совсем недавно. Речь идет о предъявленном в [14] примере уравнения (1) без инерциального $C^{1}$-многообразия $\mathscr{M} \subset X^{\alpha}$. Построить аналогичный контрпример с заменой класса гладкости $\mathscr{M}$ на Lip пока не удается.

Важно упомянуть весьма интересные работы Камаева [15], [16], в которых дана конструкция инвариантного $C^{1}$-непрерывного семейства гладких устойчивых многообразий конечной коразмерности для аттрактора уравнений типа (2) и соответствуюших систем уравнений. Было бы полезно выявить в общем случае зависимость между наличием такого семейства многообразий и конечномерностью предельной динамики эволюционных задач (1).

Здесь не представлены количественные оценки фазовой размерности ОДУ, описывающего предельную динамику (1), и их сравнение с известными оценками размерности аттрактора или же инерциального многообразия (если таковое имеется). Эта несомненно перспективная тема выходит за рамки настоящей публикации и может стать предметом дальнейших исследований.

\section{§1. Топологические условия}

Уточним ряд понятий, связанных с уравнением (1), и напомним некоторые известные свойства таких уравнений (см., в частности, [2]).

Пусть $X$ - бесконечномерное сепарабельное банахово пространство с нормой $|\cdot|$ и $\sigma(\cdot),\|\cdot\|, R(\lambda ; \cdot)$ - спектр, норма, резольвента линейных операторов в $X$. Замкнутый линейный оператор $A$ в (1) с плотной областью определения $\mathscr{D}(A) \subset X$ предполагаем секториальным и дискретным. Первое означает, что при надлежащих $k>0$ и $\lambda_{0} \in \mathbb{R}$ спектр $\sigma(A)$ лежит в секторе $|\operatorname{Im} \lambda|<k \operatorname{Re}\left(\lambda-\lambda_{0}\right)$ комплексной плоскости $\mathbb{C}$, а вне его

$$
\|R(\lambda ; A)\| \leqslant M /\left(1+\left|\lambda-\lambda_{0}\right|\right), \quad M=M\left(A, k, \lambda_{0}\right) .
$$

Дискретность $A$ понимается как компактность $R(\lambda ; A)$. Считаем, в дальнейшем, что $\lambda_{0}=0$, или, эквивалентно, $\operatorname{Re} \sigma(A)>0$ (т.е. $\operatorname{Re} \lambda>0$ на $\sigma(A)$ ). Это позволяет корректно определить для всех $\theta \in \mathbb{R}$ степени $A^{\theta}$ и банаховы пространства $X^{\theta}=$ $\mathscr{D}\left(A^{\theta}\right)$ с нормой $|u|_{\theta}=\left|A^{\theta} u\right|$. При $\theta<0$ операторы $A^{\theta}$ вполне непрерывны в $X$ и $\mathscr{D}\left(A^{\theta}\right)$ есть пополнение $X$ по норме $|\cdot|_{\theta}$. Кроме того: $X^{0}=X$ и $X^{1}=\mathscr{D}(A)$; для $\beta<\theta$ вложения $X^{\theta} \subset X^{\beta}$ вполне непрерывны; при $\beta, \theta \in \mathbb{R}$ операторы $A^{\beta}$ изометрично отображают $X^{\theta+\beta}$ на $X^{\theta}$.

Используем обозначения $B C^{\nu}\left(Y_{1}, Y_{2}\right), \nu \in \mathbb{Z}^{+}$, или $B C\left(Y_{1}, Y_{2}\right)$ для классов ограниченных на шарах $C^{\nu}$-гладких или непрерывных отображений $\Pi: Y_{1} \rightarrow Y_{2}$ пространств Банаха $Y_{1}, Y_{2}$.

Если $\alpha \in[0,1)$ и $F \in B C^{2}\left(X^{\alpha}, X\right)$, то отвечающая (1) задача Коши с $u(0)=$ $u_{0} \in X^{\alpha}$ имеет сильное локальное решение $u(t) \in C^{2}\left(\left(0, t^{*}\right), X^{\alpha}\right) \cap C\left(\left[0, t^{*}\right), X^{\alpha}\right)$ для $t^{*}=t^{*}\left(u_{0}\right)>0$, причем фактически $u(t) \in X^{1}$ на $\left(0, t^{*}\right)$. Постулируем еше диссипативность (1) в $X^{\alpha}$, т.е. глобальное по $t>0$ существование решений $u(t)=\Phi_{t} u_{0}$ и наличие такого (втягиваюшего) шара $\mathscr{B}_{0} \subset X^{\alpha}$, что $\Phi_{t} \mathscr{B} \subset \mathscr{B}_{0}$ для каждого шара $\mathscr{B} \subset X^{\alpha}$ при $t>t_{0}(\mathscr{B})$. Тогда фазовый полупоток $\left\{\Phi_{t}\right\}_{t \geqslant 0}-$ 
класса $C^{2}$ как отображение $(0, \infty) \times X^{\alpha} \rightarrow X^{\alpha}$. Для произвольного множества $\mathscr{U} \subset X^{\alpha}$ ограниченность образа $\Phi_{t} \mathscr{U}$ в $X^{\theta}$ при $t>0, \alpha<\theta<1$ (а значит, и относительная компактность $\Phi_{t} \mathscr{U}$ в $\left.X^{\alpha}\right)$ следует из $X^{\alpha}$-ограниченности трубки тока $\bigcup_{0 \leqslant \tau \leqslant t} \Phi_{\tau} \mathscr{U}$ с помошью доводов типа [2, теорема 3.3.6]. Отсюда явствует, что эволюционные операторы $\Phi_{t}$ компактны на любом шаре $\mathscr{B} \subset X^{\alpha}$, коль скоро $t>t_{0}(\mathscr{B})$.

Итак, принимаем три основные гипотезы:

(H1) линейный оператор $A$ дискретен и секториален, спектр $\sigma(A)$ счетен и $\operatorname{Re} \sigma(A)>0$

(Н2) $F \in B C^{2}\left(X^{\alpha}, X\right)$ при некотором $\alpha \in[0,1)$;

(Н3) уравнение (1) диссипативно в $X^{\alpha}$.

Требование счетности $\sigma(A)$ является чисто техническим. В гильбертовом пространстве предположению (Н1) удовлетворяет всякий дискретный нормальный оператор со спектром в секторе $|\operatorname{Im} \lambda|<k \operatorname{Re} \lambda$ при $k=$ const $>0$, например любой дискретный положительно определенный оператор.

Обозначаем через $G(u)$ векторное поле $F(u)-A u$ уравнения (1). Множество $\mathscr{U} \subset X^{\alpha}$ инвариантно, если $\Phi_{t} \mathscr{U}=\mathscr{U}$ для $t>0$ (так что, на самом деле, $\left.\mathscr{U} \subset X^{1}\right)$. Ограниченные инвариантные подмножества $X^{\alpha}$ относительно компактны. В условиях (H1)-(Н3) фазовый полупоток $\left\{\Phi_{t}\right\}$ обладает (см. [8]-[10]) компактным аттрактором $\mathscr{A}$ - максимальным ограниченным инвариантным подмножеством $X^{\alpha}$. Как показано в $[1, \S 4]$, функция $G: \mathscr{A} \rightarrow X^{\alpha}$ гёльдерова в $X^{\alpha}$-метрике и $|u|_{1} \leqslant$ const на $\mathscr{A}$. Последнее справедливо и при $F \in B C^{1}\left(X^{\alpha}, X\right)$, что доказывается без труда, если перейти к интегральной форме записи в (1). Непосредственно из [1, теорема 1.4] следует

Лемма 1.1. Функиия $A: \mathscr{A} \rightarrow X$ гёльдерова в $X^{\alpha}$-метрике.

ЗАмечАниЕ 1.2. Гипотеза (Н1) выполняется одновременно для операторов $A: X^{1} \rightarrow X$ и $A: X^{1+\beta} \rightarrow X^{\beta}$ с $\beta>0$. Таким образом, заменив в (Н2), (Н3) пару пространств $\left(X^{\alpha}, X\right)$ на $\left(X^{\alpha+\beta}, X^{\beta}\right)$, можно перенести все вышеперечисленные свойства динамики (1) на фазовое пространство $X^{\alpha+\beta}$. То же верно и в контексте последуюших построений.

Наличие втягивающего шара для полупотока $\left\{\Phi_{t}\right\}$ необходимо только как гарантия существования компактного аттрактора.

ЗАМЕЧАНИЕ 1.3. Если ограничиться изучением динамики (1) на произвольных инвариантных компактах $\mathscr{K} \subset X^{\alpha}$, то вместо (Н3) достаточно постулировать продолжимость решений $u(t)$ на $(0, \infty)$ при всех начальных значениях $u_{0} \in X^{\alpha}$.

Оба замечания относятся в полной мере и к результатам [1].

Для $a>0$ обозначим через $\mathscr{P}_{a}$ конечномерньй спектральньй проектор оператора $A$ в $X$, соответствующий части спектра с $\operatorname{Re} \lambda<a$. Проекторы $\mathscr{P}_{a}$ коммутируют с $A^{\alpha}$ и непрерывны в $X$ и $X^{\alpha}$.

Скажем (см. [1, определение 1.1]), что фазовая динамика (1) на инвариантном компакте $\mathscr{K} \subset X^{\alpha}$ конечномерна, если найдутся ОДУ с липшицевым векторным полем и разрешаюшим потоком $\left\{\varphi_{t}\right\}$ в $\mathbb{R}^{n}$, а также липшицево вложение $\Psi: \mathscr{K} \rightarrow$ $\mathbb{R}^{n}$ такие, что

$$
\Psi \Phi_{t} u=\varphi_{t} \Psi u, \quad u \in \mathscr{K}, \quad t \geqslant 0 .
$$


При $\mathscr{K}=\mathscr{A}$ в том же смысле говорится о конечномерности предельной динамики уравнения (1).

Согласно [1, теорема 1.6] следующие утверждения равносильны:

(FD) фазовая динамика на $\mathscr{K}$ конечномерна;

$(\mathrm{VF})|G(u)-G(v)|_{\alpha} \leqslant c|u-v|_{\alpha}$ для $u, v \in \mathscr{K}, c=c(\mathscr{K})$;

$(\mathrm{Fl})$ полупоток $\left\{\Phi_{t}\right\}$ на $\mathscr{K}$ инъективен и расширяется до липшицева в $X^{\alpha}$-метрике потока;

$(\mathrm{GrF})$ при некотором $a>0$ имеем оценку $|u-v|_{\alpha} \leqslant c\left|\mathscr{P}_{a}(u-v)\right|_{\alpha}$ для $u, v \in \mathscr{K}$, $c=c(\mathscr{K}, a)$;

$(\mathrm{Gr})$ найдется конечномерный непрерьвный в $X^{\alpha}$ (и в $X^{\alpha-1}$, если $X$ нерефлексивно) проектор $P$ такой, что $|u-v|_{\alpha} \leqslant c|P(u-v)|_{\alpha}$ при $u, v \in \mathscr{K}, c=c(\mathscr{K}, P)$;

(ЕМ) метрики $X^{\alpha}$ и $X^{\alpha-1}$ на $\mathscr{K}$ эквивалентны.

Эта теорема устанавливает логический цикл $(\mathrm{VF}) \rightarrow(\mathrm{Fl}) \rightarrow(\mathrm{GrF}) \rightarrow(\mathrm{Gr}) \rightarrow$ $(\mathrm{EM}) \rightarrow(\mathrm{VF})$ и импликации $(\mathrm{FD}) \rightarrow(\mathrm{Fl}),(\mathrm{Gr}) \rightarrow(\mathrm{FD})$. При этом переход $(\mathrm{Fl}) \rightarrow(\mathrm{GrF})$ требует дополнительного условия [1, предположение 1.4] на оператор $A$ в терминах характеристик полугрупшы $\{\exp (-t A)\}_{t \geqslant 0}$.

Сформулируем еще два критерия конечномерности динамики на инвариантных компактах.

TEOPEMA 1.4. Пусmb

$$
\lim _{a \rightarrow \infty}\left|u-\mathscr{P}_{a} u\right|_{\alpha}=0
$$

при всех $u \in X^{\alpha}$ и $\mathscr{K}$ - инвариантный компакт уравнения (1) в $X^{\alpha}$. Тогда каждое из следующих утверждений равносильно конечномерности динамики на $\mathscr{K}$ :

(KC) множество $\mathscr{K}^{0}$ точек вида $w=(u-v) /|u-v|_{\alpha} c u, v \in \mathscr{K}, \quad u \neq v$, относительно компактно в $X^{\alpha}$;

$(\mathrm{GrL})$ для всякого $w \in \mathscr{K}$ найдутся $X^{\alpha}$-окрестности $\mathscr{V} \supset w$ и конечномерный непрерывный в $X^{\alpha}$ (а такжсе в $X^{\alpha-1}$, если $X$ нерефлексивно) проектор $P$ такие, что $|u-v|_{\alpha} \leqslant c|P(u-v)|_{\alpha}$ на $\mathscr{V} \cap \mathscr{K}, c=c(\mathscr{K}, w, P)$.

Подчеркнем, что в $(\mathrm{GrL})$ ранг $P$ может зависеть от $w$.

ДокаЗАТЕЛЬСтво. Установим логическую цеп $(\mathrm{FD}) \rightarrow(\mathrm{KC}) \rightarrow(\mathrm{GrL}) \rightarrow(\mathrm{FD})$. Поскольку, как мы знаем, $(\mathrm{FD}) \rightarrow(\mathrm{Fl}),(\mathrm{GrF}) \rightarrow(\mathrm{Gr}) \rightarrow(\mathrm{FD}),(\mathrm{EM}) \rightarrow(\mathrm{Fl})$ и, очевидно, $(\mathrm{Gr}) \rightarrow(\mathrm{GrL}), \quad$ то достаточно получить импликации $(\mathrm{Fl}) \rightarrow(\mathrm{KC}) \rightarrow(\mathrm{GrF})$ и $(\mathrm{GrL}) \rightarrow(\mathrm{EM})$.

Покажем, что (Fl) влечет (KC). Пусть $\alpha<\theta<1$. При $t>0$ верна (см. [17, лемма 5.2]) оценка $\left|\Phi_{t} u-\Phi_{t} v\right|_{\theta} \leqslant c_{t}|u-v|_{\alpha}$ на $\mathscr{K}$ с $c_{t}=c(\mathscr{K}, \theta ; t)$. Отсюда находим, пользуясь свойством (Fl) полупотока $\left\{\Phi_{t}\right\}$, что

$$
|u-v|_{\theta} \leqslant c_{1}\left|\Phi_{-1} u-\Phi_{-1} v\right|_{\alpha} \leqslant N|u-v|_{\alpha}, \quad N=\text { const } .
$$

Итак, $|(u-v) /| u-\left.\left.v\right|_{\alpha}\right|_{\theta} \leqslant N$ на $\mathscr{K}$, множество $\mathscr{K}^{0}$ ограничено в $X^{\theta}$ и, стало быть, относительно компактно в $X^{\alpha}$.

Установим импликацию $(\mathrm{KC}) \rightarrow(\mathrm{GrF})$. Из (3) явствует, что $\left\|I-\mathscr{P}_{a}\right\|_{\alpha} \leqslant$ const $^{1}$, следовательно (по теореме Арцела), сходимость проекторов $\mathscr{P}_{a} \rightarrow I$ при $a \rightarrow \infty$

\footnotetext{
${ }^{1}$ Здесь и всюду далее $I=\mathrm{id}$.
} 
равномерна на относительно компактном множестве $\mathscr{K}^{0} \subset X^{\alpha}$ и $\left|h-\mathscr{P}_{a} h\right|_{\alpha} \leqslant$ $\varepsilon_{a}|h|_{\alpha} \mathrm{c} \varepsilon_{a} \rightarrow 0, h=u-v$ для $u, v \in \mathscr{K}$. Если взять $\varepsilon_{a}<1, c=\left(1-\varepsilon_{a}\right)^{-1}$, то $\left|h-\mathscr{P}_{a} h\right|_{\alpha} \leqslant c \varepsilon_{a}\left|\mathscr{P}_{a} h\right|_{\alpha}$ и $|u-v|_{\alpha} \leqslant c\left|\mathscr{P}_{a}(u-v)\right|_{\alpha}$ на $\mathscr{K}$.

Осталось показать, что $(\mathrm{GrL}) \rightarrow(\mathrm{EM})$. Пусть $\delta>0$ - число Лебега [18, п. 2.13.4] открытого (будем считать конечного) покрытия компакта $\mathscr{K}$ множествами $\mathscr{U}(w)=$ $\mathscr{V} \cap \mathscr{K}$; тогда любая пара точек $u, v \in \mathscr{K}$ с $|u-v|_{\alpha}<\delta$ лежит в одном из $\mathscr{U}(w)$. Действуя, как при выводе импликации $(\mathrm{Gr}) \rightarrow(\mathrm{EM})$ в [1, теорема 1.6], приходим к оценке $|u-v|_{\alpha} \leqslant c|u-v|_{\alpha-1}$ на $X^{\alpha}$-замыкании каждого из множеств $\mathscr{U}(w)$ с единой константой $c>0$. Для $u, v \in \mathscr{K}$ с $|u-v|_{\alpha} \geqslant \delta$ аналогичное соотношение также справедливо, иначе мы имели бы абсурдную ситуацию существования таких сходящихся в $X^{\alpha}$-метрике последовательностей $\left\{u_{l}\right\},\left\{v_{l}\right\} \subset \mathscr{K}$, что $\left|u_{l}-v_{l}\right|_{\alpha} \geqslant \delta$, но $\left|u_{l}-v_{l}\right|_{\alpha-1} \rightarrow 0$ при $l \rightarrow \infty$. Наконец, противоположное неравенство $|u-v|_{\alpha} \geqslant c^{\prime}|u-v|_{\alpha-1}$ на $\mathscr{K}$ следует из непрерьвности вложения $X^{\alpha} \subset X^{\alpha-1}$. Теорема 1.4 доказана полностью.

Теперь у нас есть расширенный логический цикл $(\mathrm{VF}) \rightarrow(\mathrm{Fl}) \rightarrow(\mathrm{KC}) \rightarrow(\mathrm{GrF}) \rightarrow$ $(\mathrm{Gr}) \rightarrow(\mathrm{GrL}) \rightarrow(\mathrm{EM}) \rightarrow(\mathrm{VF})$ наряду с импликациями $(\mathrm{FD}) \rightarrow(\mathrm{Fl})$ и $(\mathrm{Gr}) \rightarrow(\mathrm{FD})$, где предположение (3) использовано лишь в переходе $(\mathrm{KC}) \rightarrow(\mathrm{GrF})$. По существу, (3) означает базисность в $X^{\alpha}$ конечномерных инвариантных подпространств оператоpa $A$, отвечаюших упорядоченным по возрастанию $\operatorname{Re} \lambda$ спектральным множествам $\{\lambda \in \sigma(A): \operatorname{Re} \lambda=$ const $\}$, и заведомо выполнено, коль скоро $A$ - спектральньй [19] оператор в $X$, а значит, и в $X^{\alpha}$. Когда пространство $X$ гильбертово, в этом качестве подходят, скажем, операторы $A$, подобные нормальным.

Перейдем к обсуждению условия конечномерности фазовой динамики на инвариантном компакте $\mathscr{K} \subset X^{\alpha}$, представляющего особый интерес. Теорема 1.5 работы [1] устанавливает связь между вложимостью $\mathscr{K}$ в конечномерное (достаточно регулярное) подмногообразие $\mathscr{M} \subset X^{\alpha}$ и конечномерностью динамики на $\mathscr{K}$. Здесь данному утверждению будет придана форма, близкая к окончательной по порядку гладкости $\mathscr{M}$.

ТЕОРема 1.5. Пусть $\mathscr{K}$ - инвариантный компакт уравнения (1) в $X^{\alpha} u$ для оператора А справедливо соотношение (3). Если $\mathscr{K} \subset \mathscr{M}$, где $\mathscr{M}$ - конечномерное $C^{1}$-подмногообразие в $X^{\alpha}$, то фазовая динамика на $\mathscr{K}$ конечномерна. Обратно, если динамика на $\mathscr{K}$ конечномерна, то найдется такое $a>0$, что множество Ж принадлежит графику некоторой равномерно липиичевой функиии, действующей из $\mathscr{P}_{a} X^{\alpha}{ }_{\boldsymbol{\theta}}\left(I-\mathscr{P}_{a}\right) X^{\alpha}$.

Эта теорема анонсирована в [20]. Отличие ее от упомянутого результата [1] состоит в понижении требуемого класса гладкости $\mathscr{M}$ с $C^{2}$ до $C^{1}$ и нюансах в ограничениях на линейный оператор $A$. Как видим, предельная динамика (1) конечномерна, коль скоро аттрактор $\mathscr{A}$ можно вложить в конечномерное $C^{1}$-многообразие $\mathscr{M} \subset X^{\alpha}$.

ДокАЗАТЕЛЬСТво. Вторая (обратная) часть теоремы доказывается так же, как в [1]. Уточним только, что необходимая для этого импликация $(\mathrm{FD}) \rightarrow(\mathrm{GrF})$ верна при условии (3) на оператор $A$.

Далее исходим из включения $\mathscr{K} \subset \mathscr{M}$. Конечномерное $C^{1}$-многообразие $\mathscr{M}$ в банаховом пространстве $X^{\alpha}$ локально устроено как график гладкой функции над касательным подпространством. Поэтому для каждой точки $w \in \mathscr{M}$ найдутся 
непрерывный в $X^{\alpha}$ проектор $P$ ранга $n=\operatorname{dim} \mathscr{M}$, постоянная $c=c(\mathscr{M}, w, P)$ и замкнутый шар $\mathscr{V}=\left\{u \in X^{\alpha}:|u-w|_{\alpha} \leqslant \varepsilon\right\}$ с $\varepsilon=\varepsilon(w)$ такие, что $|u-v|_{\alpha} \leqslant$ $c|P(u-v)|_{\alpha}$ на $\mathscr{M}(w)=\mathscr{V} \cap \mathscr{M}$. Ясно, что $\mathscr{M}(w)$ - компактное $C^{1}$-многообразие размерности $n$. Пользуясь [1, лемма 2.3], выберем непрерывный в $X^{\alpha}$ и $X^{\alpha-1}$ проектор $P_{0}$ ранга $n$ таким образом, чтобы

$$
\left|\left(P-P_{0}\right)(u-v)\right|_{\alpha} \leqslant(2 c)^{-1}|u-v|_{\alpha}, \quad u, v \in \mathscr{M}(w) .
$$

Отсюда без труда получаем оценку $|u-v|_{\alpha} \leqslant 2 c\left|P_{0}(u-v)\right|_{\alpha}$ на $\mathscr{M}(w)$. Тем самым, инвариантный компакт $\mathscr{K}$ обладает свойством (GrL), и по теореме 1.4 фазовая динамика на $\mathscr{K}$ конечномерна. Доказательство завершено.

ЗАмЕчАниЕ 1.6. Если предельная динамика уравнения (1) конечномерна, то периоды его периодических решений ограничены снизу положительным числом.

Это есть следствие определения конечномерной динамики на аттракторе и известной оценки снизу [21] периодов периодических решений ОДУ в $\mathbb{R}^{n}$ через константу Липшища соответствуюшего векторного поля.

\section{§2. Аналитический подход}

Главная цель данного параграфа - нахождение конструктивных условий на коэффищиенты уравнения (1) в гильбертовом пространстве $X$, влекуших свойство $(\mathrm{GrL})$ аттрактора $\mathscr{A} \subset X^{\alpha}$, а значит, и конечномерность динамики на $\mathscr{A}$. Полученные условия будут затем использованы в $\S 3$ для доказательства конечномерности предельной динамики параболических уравнений (2). Ряд необходимых для изложения вспомогательных результатов вынесен в $\S 4$.

Там, где не указано иное, пространство $X$ по-прежнему банахово. Введем обозначения: $\mathscr{N}=\mathscr{A} \times \mathscr{A} ; D$ - дифференцирование $\Phi$ реше; $\mathscr{L}\left(X^{\theta}, X^{\beta}\right)$ - пространство непрерывных линейных операторов, действующих из $X^{\theta}$ в $X^{\beta}$, и $\mathscr{L}\left(X^{\theta}\right)=$ $\mathscr{L}\left(X^{\theta}, X^{\theta}\right) ;\|\cdot\|,\|\cdot\|_{\alpha}-$ нормы в $\mathscr{L}(X), \mathscr{L}\left(X^{\alpha}\right)$ и $\|\cdot\|_{\alpha, 0},\|\cdot\|_{0, \alpha}-$ нормы в $\mathscr{L}\left(X^{\alpha}, X\right), \mathscr{L}\left(X, X^{\alpha}\right)$. Объектом нашего внимания станут векторные (в основном операторные) поля П $(u, v)$ на $\mathscr{N}$ со значениями в различных пространствах Банаха $Y$. Множество $\mathscr{N}$ снабдим метрикой, индуцированной из $X^{\alpha} \times X^{\alpha}$.

ОПРЕДЕЛЕНИЕ 2.1. Непрерывное поле П: $\mathscr{N} \rightarrow Y$ называем регулярнылм, если для любых $u, v \in \mathscr{A}$ функция $\Pi\left(\Phi_{t} u, \Phi_{t} v\right):[0, \infty) \rightarrow Y-$ класса $C^{1}$ с равномерно по $u, v$ ограниченной производной в нуле $\partial_{t} \Pi(u, v)$.

Из гладкости полупотока $\left\{\Phi_{t}\right\}$ и инвариантности компакта $\mathscr{A} \subset X^{\alpha}$ следует регулярность тождественного вложения $\mathcal{N} \rightarrow X^{\alpha} \times X^{\alpha}$, а стало быть, и регулярность всякого поля П: $\mathcal{N} \rightarrow Y$, продолжимого до $C^{1}$-отображения в $\left(X^{\alpha} \times\right.$ $\left.X^{\alpha}\right)$-окрестность множества $\mathcal{N}$. При этом

$$
\partial_{t} \Pi(u, v)=D \Pi(u, v)\left(\partial_{t} u, \partial_{t} v\right), \quad \partial_{t} u=G(u)=F(u)-A u
$$

Действуем далее по плану, намеченному во введении. Для $u, v \in \mathscr{A}$ полагаем

$$
\begin{aligned}
& T(u, v)=T_{0}(u, v)+\int_{0}^{1} D F(\tau u+(1-\tau) v) d \tau \\
& B(u, v)=\omega I+A-T(u, v)
\end{aligned}
$$


где $T_{0}$ - произвольное (пока) поле операторов, ограниченное со значениями в $\mathscr{L}\left(X^{\alpha}\right)$ и регулярное со значениями в $\mathscr{L}\left(X^{\alpha}, X\right)$, а $\omega>0$ - числовой параметр. Здесь $T=T_{0}+T_{1}, T_{1}(u, u)=D F(u)$, и поскольку $F \in C^{2}\left(X^{\alpha}, X\right)$, то $T_{1}(u, v) \in \mathscr{L}\left(X^{\alpha}, X\right)$. Поле $T_{1}$ продолжается с $\mathscr{N}$ до $C^{1}$-отображения $X^{\alpha} \times$ $X^{\alpha} \rightarrow \mathscr{L}\left(X^{\alpha}, X\right)$, откуда вытекает регулярность поля $T: \mathscr{N} \rightarrow \mathscr{L}\left(X^{\alpha}, X\right)$. Операторы $T_{0}(u, v)$ в (4a) играют роль искусственной поправки, призванной “улучшить" свойства поля $B(u, v)$. Заметим, что

$$
\mathscr{D}(B(u, v)) \equiv \mathscr{D}(A)=X^{1}, \quad B(u, v) \in \mathscr{L}\left(X^{1}, X\right)
$$

и по интегральной теореме о среднем значении

$$
G(u)-G(v)=\left(B_{0}(u, v)-B(u, v)\right)(u-v), \quad B_{0}(u, v)=\omega I-T_{0}(u, v) .
$$

Пусть

$$
\begin{array}{rlrl}
\Sigma & =\bigcup_{u, v \in \mathscr{A}} \sigma(B(u, v)), & \mathscr{R} & =\mathbb{C} \backslash \Sigma, \\
\Gamma_{a} & =\{\lambda \in \mathbb{C}: \operatorname{Re} \lambda=a\}, \quad \Gamma(a, \xi)=\{\lambda \in \mathbb{C}: a-\xi \leqslant \operatorname{Re} \lambda \leqslant a+\xi\}
\end{array}
$$

при $a>\xi>0$. Число $\omega$ в (4б) выбирается так (см. ниже лемму 4.1), чтобы обеспечить соотношение $\operatorname{Re} \Sigma>0$ для $\Sigma=\Sigma(\omega)$. Если $\Gamma_{a} \subset \mathscr{R}$, то обозначаем символом $P_{a}(u, v)$ непрерывный в $X$ спектральный проектор оператора $B(u, v)$, отвечающий части спектра с $\operatorname{Re} \lambda<a$, и записываем $Q_{a}(u, v)=I-P_{a}(u, v)$. Согласно $[2 ; \S 1.4,1.5]$ операторы $B(u, v)$ дискретны и секториальны в $X, \mathscr{D}\left(B^{\alpha}\right)=$ $X^{\alpha}$, степени $B^{\alpha}, B^{-\alpha}$ коммутируют с $P_{a}, Q_{a}$ и резольвентой $R(\lambda ; B)$. Проекторы $P_{a}(u, v)$ имеют конечный ранг $n=n(a)$ для всех $u, v \in \mathscr{A}$. Кроме того, $P_{a}, Q_{a} \in$ $\mathscr{L}\left(X^{\alpha}\right)$, а формулируемые далее леммы $4.2,4.3$ гарантируют регулярность полей операторов $P_{a}, Q_{a}: \mathscr{N} \rightarrow \mathscr{L}\left(X^{\alpha}\right), B^{\alpha}: \mathscr{N} \rightarrow \mathscr{L}\left(X^{\alpha}, X\right), B^{-\alpha}: \mathscr{N} \rightarrow \mathscr{L}\left(X, X^{\alpha}\right)$. Регулярность поля $P_{a}$ имеет следствием оценку

$$
\left\|\partial_{t} P_{a}(u, v)\right\|_{\alpha} \leqslant m(a)
$$

на $\mathscr{N}$ с $m(a)<\infty$. Сформулируем еше одно важное понятие.

ОПРЕДЕЛЕНИЕ 2.2. В случае гильбертова пространства $X$ будем говорить, что поле $B: \mathscr{N} \rightarrow \mathscr{L}\left(X^{1}, X\right)$ равномерно скалярного типа, если $B(u, v)=$ $S^{-1}(u, v) H(u, v) S(u, v)$ на $\mathscr{N}$, где линейные операторы $H(u, v)$ нормальны в $X$, поле $S: \mathscr{N} \rightarrow \mathscr{L}(X)$ регулярно, а поле $S^{-1}: \mathscr{N} \rightarrow \mathscr{L}(X)$ ограничено.

В данной формулировке $B(u, v)$ - операторы скалярного типа (см. [19]) при всех $u, v \in \mathscr{A}$. Для случая $T_{0}=0, u=v$ представление $B=S^{-1} H S$ из определения 2.2 фактически использовалось Камаевым [15], [16] в связи с изучением фазовой динамики вблизи аттрактора уравнений (скалярных или векторных) чуть более широкого класса, чем (2). В цитированных работах к линеаризации правой части уравнения применялось либо обычное преобразование Лиувилля, либо [16] некоторая его модификация.

В контексте определения 2.2 отметим неравенства

$$
\|S(u, v)\| \leqslant \gamma, \quad\left\|S^{-1}(u, v)\right\| \leqslant \gamma, \quad\left\|\partial_{t} S(u, v)\right\| \leqslant \gamma_{1}
$$


на $\mathscr{N}$ с $\gamma, \gamma_{1}=$ const. Ясно, что $\sigma(B)=\sigma(H)$ и $P_{a}^{\prime}=S P_{a} S^{-1}, Q_{a}^{\prime}=S Q_{a} S^{-1}$ ортогональные спектральные проекторы нормальных операторов $H$. Подчеркнем, что область определения $\mathscr{D}(H)=S \mathscr{D}(B)=S X^{1}$ зависит, вообще говоря, от $u, v$.

Если $X$ - гильбертово пространство со скалярным произведением $(\cdot, \cdot)$ и при каком-то $a>\omega$ прямая $\Gamma_{a}$ лежит в $\mathscr{R}$, то для $u, v \in \mathscr{A}, h=u-v$ принимаем

$$
\begin{gathered}
p=S(u, v) B^{\alpha}(u, v) P_{a}(u, v) h, \quad q=S(u, v) B^{\alpha}(u, v) Q_{a}(u, v) h, \\
W_{a}(u, v)=\frac{1}{2}\left(|q|^{2}-|p|^{2}\right) .
\end{gathered}
$$

Векторные поля $p(u, v), q(u, v)$ регулярны со значениями в $X$ (как комбинации регулярных полей), а значит, регулярно и числовое поле $W_{a}(u, v)$. Существенным в дальнейшем оказьвается следуюшее предположение о динамике уравнения (1):

$$
\partial_{t} W_{a}(u, v)+2(a-\omega) W_{a}(u, v) \leqslant 0
$$

для $u, v \in \mathscr{A}$. Поскольку $Q_{a}^{\prime} S=S Q_{a}, Q_{a} B^{\alpha}=B^{\alpha} Q_{a}$ и $Q_{a}^{2}=Q_{a}$, то

$$
Q_{a}^{\prime} q=Q_{a}^{\prime} S B^{\alpha} Q_{a} h=S B^{\alpha} Q_{a} h=q
$$

Точно так же $P_{a}^{\prime} p=p$, и ортогональность проекторов $P_{a}^{\prime}, Q_{a}^{\prime}$ приводит к равенству $(p, q)=0$. Соотношение $(7)$ есть нелинейный аналог сходного по форме требования [13, теорема 5], позволяющего построить инерциальное многообразие для уравнения (1) с самосопряженной линейной частью. При $|q| \geqslant|p|$ и соответственно при $|q| \leqslant|p|$ неравенство (7) дает нелинейные обобщения хорошо известных в теории эволюционных систем "свойства сдавливания" и "условия конуса" [10, гл. 8].

Пусть $L, N$ - зависяшие лишь от $A, F, T_{0}$ постоянные из лемм 4.1, 4.3 (см. ниже) и $\left\|T_{0}(u, v)\right\|_{\alpha} \leqslant K$ на $\mathscr{N}$.

Теорема 2.3. Допустим, что пространство $X$ гильбертово, оператор $A$ обладает свойством (3) $u \operatorname{Re} \Sigma>0$ для $\Sigma=\Sigma(\omega)$. Предположим, что:

а) $B: \mathscr{N} \rightarrow \mathscr{L}\left(X^{1}, X\right)$ есть поле операторов равномерно скалярного типа;

б) множсество $\mathscr{R}$ содержит полосу $\Gamma(a, \xi)$ с а $>\omega, \xi \geqslant \gamma \gamma_{1}+\gamma^{2} N+$ $\gamma^{2} L^{2}(K+m(a))$.

Тогда справедливо соотношение (7) и предельная динамика уравнения (1) конечномерна.

Заметим, что в оценке $\xi$ лишш величина $m$ зависит от $a$. Ключевым для вывода теоремы является следуюшее утверждение.

ЛЕмма 2.4. Условия теоремы 2.3 влекут неравенство (7).

ДоказАтЕЛьство. Опускаем в обозначениях векторных полей на $\mathscr{N}$ зависимость от $u, v \in \mathscr{A}$ и, как правило, от $a$. Например, $P_{a}(u, v)=P, Q_{a}(u, v)=Q$. Для регулярных полей операторов на $\mathcal{N}$ записываем, кратко, $\partial_{t} S(u, v)=S_{t}$ и т.д. Если $h=u-v$, то $\partial_{t} h=\partial_{t} u-\partial_{t} v=\omega h-T_{0} h-B h$. При этом $h \in X^{1}$, а поскольку $\partial_{t} h=G(u)-G(v) \in X^{\alpha}$ и $T_{0} h \in X^{\alpha}$, то $B h \in X^{\alpha}$. Но $X^{\alpha}=\mathscr{D}\left(B^{\alpha}\right)$, так что ${ }^{2}$ $h \in \mathscr{D}\left(B^{1+\alpha}\right)$ и $B^{\alpha} h \in X^{1}$.

\footnotetext{
${ }^{2} h \in \mathscr{D}\left(B^{2}\right)$ при $T_{0} \in \mathscr{L}\left(X^{1}\right)$, в частности при $T_{0}=0$.
} 
Исходим из выражения $\partial_{t} W_{a}=\left(\partial_{t} q, q\right)-\left(\partial_{t} p, p\right)$ с $q=S B^{\alpha} Q h, p=S B^{\alpha} P h$, $p+q=S B^{\alpha} h$. Преобразуем сначала величину $\left(\partial_{t} q, q\right)$. Положим $V=S B^{\alpha} Q$ и $U=B^{-\alpha} S^{-1}$; тогда $q=V h, h=U(p+q), \partial_{t} q=V_{t} h+V \partial_{t} h$ или, подробнее,

$$
\partial_{t} q=\left(V_{t} U+\omega V U-V T_{0} U-V B U\right)(p+q) .
$$

Будем пользоваться равенствами $Q B^{\alpha}=B^{\alpha} Q, Q B^{-\alpha}=B^{-\alpha} Q, B=S^{-1} H S$ и $Q=S^{-1} Q^{\prime} S$, а также $Q^{\prime} p=0, Q^{\prime} q=q$. Как видим, $S B^{\alpha} h \in S X^{1}=\mathscr{D}(H)$, значит, $q=S B^{\alpha} Q h=Q^{\prime} S B^{\alpha} h \in \mathscr{D}(H)$ и $p=S B^{\alpha} h-q \in \mathscr{D}(H)$. Легко находим, что $V U(p+q)=q, V B U(p+q)=H q, V T_{0} U=Q^{\prime} U_{1}$ с $U_{1}=S B^{\alpha} T_{0} B^{-\alpha} S^{-1}$. Далее, $Q_{t}+P_{t}=0$ и

$$
V_{t}=S_{t} B^{\alpha} Q+S\left(B^{\alpha}\right)_{t} Q-S B^{\alpha} P_{t}=J_{1}+J_{2}-J_{3} .
$$

Элементарные выкладки приводят к формулам: $J_{1} U=V_{1} Q^{\prime}$, где $V_{1}=S_{t} S^{-1}$; $J_{2} U=V_{2} Q^{\prime}$ с $V_{2}=S\left(B^{\alpha}\right)_{t} B^{-\alpha} S^{-1} ; J_{3} U=V_{3}$ с $V_{3}=S B^{\alpha} P_{t} B^{-\alpha} S^{-1}$. Таким образом, $\left(J_{1} U\right)(p+q)=V_{1} q,\left(J_{2} U\right)(p+q)=V_{2} q$ и

$$
\left(\partial_{t} q, q\right)=\left(\left(V_{1}+V_{2}\right) q, q\right)-\left(V_{3}(p+q), q\right)-\left(U_{1}(p+q), q\right)+((\omega I-H) q, q) .
$$

Мы воспользовались тем, что $\left(Q^{\prime}\right)^{*}=Q^{\prime}$ в $X$ и, следовательно, $\left(Q^{\prime} U_{1}(p+q), q\right)=$ $\left(U_{1}(p+q), q\right)$.

Действуя вполне аналогично, приходим к выводу, что

$$
\left(\partial_{t} p, p\right)=\left(\left(V_{1}+V_{2}\right) p, p\right)+\left(V_{3}(p+q), p\right)-\left(U_{1}(p+q), p\right)+((\omega I-H) p, p) .
$$

Из неравенств (6) и неравенства (22), приведенного в приложении, явствуют оценки $\left\|V_{1}\right\| \leqslant \gamma \gamma_{1},\left\|V_{2}\right\| \leqslant \gamma^{2} N$. Кроме того, $\left\|V_{3}\right\| \leqslant \gamma^{2}\left\|B^{\alpha} P_{t} B^{-\alpha}\right\|$. Очевидные тождества

$$
\begin{gathered}
\left\|B^{\alpha} P_{t} B^{-\alpha}\right\|=\left\|A^{-\alpha} B^{\alpha} P_{t} B^{-\alpha} A^{\alpha}\right\|_{\alpha}, \quad\left\|A^{-\alpha} B^{\alpha}\right\|_{\alpha}=\left\|B^{\alpha} A^{-\alpha}\right\|, \\
\left\|B^{-\alpha} A^{\alpha}\right\|_{\alpha}=\left\|A^{\alpha} B^{-\alpha}\right\|
\end{gathered}
$$

обеспечивают с учетом (5), (6), (18) неравенство $\left\|V_{3}\right\| \leqslant \varkappa=\gamma^{2} L^{2} m(a)$. Уже отмечалось, что $(p, q)=0$, поэтому

$$
\left|\left(V_{3}(p+q), p+q\right)\right| \leqslant \varkappa\left(|p|^{2}+|q|^{2}\right) .
$$

Та же техника дает оценку $\left\|U_{1}\right\| \leqslant \varkappa_{0}=\gamma^{2} L^{2} K$ и, тем самьм,

$$
\left|\left(U_{1}(p+q), p\right)-\left(U_{1}(p+q), q\right)\right| \leqslant \varkappa_{0}|p+q| \cdot|p-q|=\varkappa_{0}\left(|p|^{2}+|q|^{2}\right) .
$$

Наконец, $(H q, q) \geqslant(a+\xi)|q|^{2}$ и $(H p, p) \leqslant(a-\xi)|p|^{2}$, так как оператор $H$ нормален, $q \in Q^{\prime} X, p \in P^{\prime} X$. Обозначая $\varkappa_{1}=\gamma \gamma_{1}+\gamma^{2} N, \varkappa_{2}=\varkappa+\varkappa_{0}$ и суммируя все сказанное, получаем в итоге

$$
\begin{aligned}
\partial_{t} W_{a}+2(a-\omega) W_{a} & =\left(\partial_{t} q, q\right)-\left(\partial_{t} p, p\right)+(a-\omega)\left(|q|^{2}-|p|^{2}\right) \\
& \leqslant \varkappa_{1}|q|^{2}+\varkappa_{2}\left(|p|^{2}+|q|^{2}\right)-\xi|q|^{2}+\varkappa_{1}|p|^{2}-\xi|p|^{2} \\
& =\left(\varkappa_{1}+\varkappa_{2}-\xi\right)\left(|p|^{2}+|q|^{2}\right) \leqslant 0,
\end{aligned}
$$

ибо $\xi \geqslant \varkappa_{1}+\varkappa_{2}$ по предположению леммы. Доказательство завершено.

Иногда ограничение на $\xi$ в теореме 2.3 можно упростить. 
ЗАмечаниЕ 2.5. Если $\alpha=0, T_{0}=0$ и операторы $B(u, v)$ нормальны, то в условии б) теоремы 2.3 имеем $\xi \geqslant m(a)$.

Здесь постоянные $\gamma_{1}, K, N$ равны нулю и $\gamma, L$ равны 1 . Подобная ситуация возникает для уравнений реакции-диффузии $u_{t}=\Delta u+f(x, u)$ в конечных областях $\mathbb{R}^{l}, l \geqslant 1$.

Лемма 2.6. Пусть пространство Х рефлексивно, $\mathscr{K}$ - инвариантный компакт уравнения (1) в $X^{\alpha}$ и оператор А удовлетворяет предположению (3). Если при некотором $a>0$ прямая $\Gamma_{a} \subset \mathscr{R} u$

$$
\left|Q_{a}(u, v)(u-v)\right|_{\alpha} \leqslant c\left|P_{a}(u, v)(u-v)\right|_{\alpha}
$$

для $u, v \in \mathscr{K}, c=c(\mathscr{K}, a)$, то фазовая динамика на $\mathscr{K}$ конечномерна.

ДоКАЗАТЕльСТво. Полагаем $P_{a}(w)=P_{a}(w, w)$ при $w \in \mathscr{K} \subset \mathscr{A}$. По лемме 4.2 поле проекторов $P_{a}: \mathscr{N} \rightarrow \mathscr{L}\left(X^{\alpha}\right)$ непрерывно в $\left(X^{\alpha} \times X^{\alpha}\right)$-метрике. Для каждого $w \in \mathscr{K}$ возьмем такую $X^{\alpha}$-окрестность $\mathscr{V} \supset w$, что

$$
\left\|P_{a}(w)-P_{a}(u, v)\right\|_{\alpha} \leqslant(2+2 c)^{-1}
$$

на $\mathscr{V} \cap \mathscr{K}$. Коль скоро $h=u-v$, то

$$
\begin{aligned}
|h|_{\alpha} & \leqslant\left|P_{a}(u, v) h\right|_{\alpha}+\left|Q_{a}(u, v) h\right|_{\alpha} \\
& \leqslant(1+c)\left|P_{a}(u, v) h\right|_{\alpha} \leqslant \frac{1}{2}|h|_{\alpha}+(1+c)\left|P_{a}(w) h\right|_{\alpha},
\end{aligned}
$$

откуда $|u-v|_{\alpha} \leqslant(2+2 c)\left|P_{a}(w)(u-v)\right|_{\alpha}$ для $u, v \in \mathscr{V} \cap \mathscr{K}$. Это означает, что компакт $\mathscr{K}$ обладает свойством $(\mathrm{GrL})$ и по теореме 1.4 динамика на $\mathscr{K}$ конечномерна. Лемма доказана.

Таким образом, получен еще один (достаточньй) критерий конечномерности динамики (1) на инвариантных компактах.

Осталось сделать совсем немного, чтобы завершить доказательство теоремы 2.3. Вновь опускаем кое-где в обозначениях зависимость от $u, v \in \mathscr{A}$ и $a>$ $\omega$. Аттрактор $\mathscr{A}$ - инвариантное множество, значит, всякое решение $u(t)$ уравнения (1) с $u(0)=u_{0} \in \mathscr{A}$ продолжается по $t$ на $\mathbb{R}$ и $u(t) \in \mathscr{A}$. Пока не утверждается, что это продолжение единственно при $t<0$. В силу тождеств $B^{\alpha} Q=Q B^{\alpha}$, $S Q=Q^{\prime} S$ и ограниченности $\mathscr{A}$ в $X^{\alpha}$ имеем

$$
2 W_{a}(u, v) \leqslant|q|^{2}=\left|S B^{\alpha} Q h\right|^{2}=\left|Q^{\prime} S B^{\alpha} h\right|^{2} \leqslant \gamma^{2} L^{2}|h|_{\alpha}^{2} \leqslant \text { const }
$$

для $u, v \in \mathscr{A}$ и $h=u-v$. Здесь использованы ортогональность проектора $Q^{\prime}$ в $X$ и неравенства $(6),(18)$. Если теперь $u_{0}, v_{0} \in \mathscr{A}, \zeta(t)=W_{a}(u(t), v(t))$ и $\lambda=2(a-$ $\omega)>0$, то из $(7)$ вытекает, что $\zeta(0) \leqslant e^{\lambda t} \zeta(t), t<0$. Но $\zeta(t) \leqslant$ const, поэтому $\zeta(0) \leqslant 0$, т.е. $W_{a}(u, v) \leqslant 0$ на $\mathcal{N}$ или же $|q| \leqslant|p|$ с $q=S B^{\alpha} Q h, p=S B^{\alpha} P h$. Опять учитьвая (6) и (18), находим

$$
|p| \leqslant \gamma\left|B^{\alpha} P h\right| \leqslant \gamma L|P h|_{\alpha}
$$

С другой стороны,

$$
|Q h|_{\alpha}=\left|A^{\alpha} Q h\right|=\left|A^{\alpha} B^{-\alpha} S^{-1} q\right| \leqslant \gamma L|q| .
$$

Отсюда выводим оценку (8) с $c=\gamma^{2} L^{2}$, по лемме 2.6 фазовая динамика на $\mathscr{A}$ конечномерна и теорема 2.3 доказана полностью. 
ЗАмЕчАниЕ 2.7. В гильбертовом случае соотношение (7) влечет оценку (8), а также неравенство $W_{a}(u, v) \leqslant 0$ на $\mathscr{N}$.

Выясним, как связано условие б) теоремы 2.3 с геометрией совокупного (по $u, v \in \mathscr{A})$ спектра $\Sigma$ операторов $B(u, v)$. При этом будет обсуждаться ситуация, когда дополнение $\mathscr{R}=\mathbb{C} \backslash \Sigma$ содержит вертикальные полосы $\Gamma(a, \xi)$ со сколь угодно большими значениями $a, \xi$. Считаем, что надлежаший выбор числа $\omega>0$ в (4б) и параметров $k>0,0 \leqslant \theta \leqslant 1$ позволяет локализовать множество $\Sigma$ в области

$$
\Omega(k, \theta)=\left\{x+i y \in \mathbb{C}: x>0,|y|<k x^{\theta}\right\} .
$$

При $\theta=1$ это всегда возможно согласно лемме 4.1. При $\theta<1$ такая локализация $\Sigma$ характерна для полулинейных параболических уравнений с частными производными в конечных областях $\mathbb{R}^{l}$.

Из условия а) теоремы 2.3 следует оценка

$$
\|R(\lambda ; B)\| \leqslant \gamma^{2} / r(\lambda), \quad B=B(u, v),
$$

где $r(\lambda)$ - расстояние ${ }^{3}$ от точки $\lambda \in \mathscr{R}$ до $\Sigma$, а $\gamma$-постоянная в (6). Действительно, $R(\lambda ; B)=S^{-1} R(\lambda ; H) S$ и $\|R(\lambda ; H)\| \leqslant 1 / r(\lambda)$ в силу нормальности операторов $H=H(u, v)$. Для $u, v \in \mathscr{A}$ и $\Gamma_{a} \subset \mathscr{R}$ лемма 4.2 дает представление

$$
\partial_{t} P_{a}(u, v)=\frac{1}{2 \pi i} \int_{a-i \infty}^{a+i \infty} E(\lambda ; u, v) d \lambda
$$

с голоморфным по $\lambda \in \mathscr{R}$ ядром $E(\lambda ; u, v) \in \mathscr{L}\left(X^{\alpha}\right)$, причем

$$
\|E(\lambda ; u, v)\|_{\alpha} \leqslant \frac{M}{r^{2}(\lambda)}\left(|\lambda|^{\alpha}+r^{\alpha}(\lambda)\right)
$$

c $M=M\left(A, F, T_{0}\right)$.

Исходя из включений $\Sigma \subset \Omega(k, \theta)$ и $\mathscr{R} \supset \Gamma(a, \xi)$ при некоторых $a>\xi>\omega$, оценим величину $m(a)$ в (5) через параметры $a, \xi, \theta$. Пусть $\chi(y)$ - такая положительная миноранта функции $r(a+i y)$ на $\mathbb{R}$, что $\chi(y) \leqslant|a+i y|$; тогда для $\lambda=a+i y$ правая часть (10) мажорируется выражением $2 M|a+i y|^{\alpha} / \chi^{2}(y)$. Если еще $\chi(y)=\chi(-y)$, то

$$
m(a) \leqslant \frac{2 M}{\pi} \int_{0}^{\infty} \frac{|a+i y|^{\alpha}}{\chi^{2}(y)} d y .
$$

Будем обозначать через $c, c_{1}, \ldots$ положительные постоянные, зависяшие лишь от $k, \theta, M$. Увеличив при необходимости числа $\omega$ и $k$, имеем $\Sigma \subset \Omega(k, \theta) \cap \Omega(k, 1)$. Значения $a, \xi$ предполагаем столь большими, сколь требуют нижеследующие рассуждения.

Начнем анализ правой части (11) со случая $\theta=0$. Полагаем $\chi(y)=\xi$ на $[0, k]$, $\chi(y)=\left(\xi^{2}+(y-k)^{2}\right)^{1 / 2}$ на $(k, \infty)$ и $\chi(y)=\chi(-y)$ при $y<0$. Понятно, что $\chi(y) \leqslant r(a+i y), \chi(y) \leqslant|a+i y|$ для всех $y \in \mathbb{R}$. Пользуясь оценками $\mid a+$

\footnotetext{
${ }^{3}$ Нетрудно показать, что $\Sigma$ замкнуто, а потому $r(\lambda)>0$.
} 
$\left.i y\right|^{\alpha} \leqslant a^{\alpha}+k^{\alpha}$ при $0 \leqslant y \leqslant k,|a+i y|^{\alpha} \leqslant a^{\alpha}+k^{\alpha}+(y-k)^{\alpha}$ при $y>k$ и производя интегрирование, получаем неравенство

$$
m(a) \leqslant c a^{\alpha} / \xi
$$

Далее, пусть $\theta=1$. Положим $\chi(y)=\xi$ на $[0,2 k a], \chi(y)=(y-k a) /\left(1+k^{2}\right)^{1 / 2}$ на $(2 k a, \infty)$ и $\chi(y)=\chi(-y)$ при $y<0$. При $y>2 k a$ величина $\chi(y)$ есть расстояние от точки $a+i y$ до границы сектора $\Omega(k, 1)$, поэтому $\chi(y) \leqslant r(a+i y), \chi(y) \leqslant|a+i y|$ на $\mathbb{R}$. Записав

$$
|a+i y|^{\alpha} \leqslant a^{\alpha}\left(1+(2 k)^{\alpha}\right)
$$

при $0 \leqslant y \leqslant 2 k a$,

$$
|a+i y|^{\alpha} \leqslant a^{\alpha}+(k a)^{\alpha}+(y-k a)^{\alpha}
$$

при $y>2 k a$ и интегрируя в (11), выводим оценку

$$
m(a) \leqslant c a^{\alpha+1} / \xi^{2}
$$

В случае $0<\theta<1$ обозначим $\nu=\theta^{-1}>1$ и $=k^{-\nu}$. Для $x_{0}>a$ и $y_{0}=k x_{0}^{\theta}$ нормаль к параболе $y=k x^{\theta}$ в $\left(x_{0}, y_{0}\right)$ пересекает прямую $x=a$ в точке с ординатой

$$
y_{1}=z\left(x_{0}\right)=y_{0}+\nu \varkappa^{2} y_{0}^{2 \nu-1}-\nu \varkappa a y_{0}^{\nu-1}
$$

или, что то же, $z\left(x_{0}\right)=k x_{0}^{\theta}+\nu k^{-1}\left(x_{0}-a\right) x_{0}^{1-\theta}$. Таким образом, $z^{\prime}\left(x_{0}\right)>0$, $y_{0}<y_{1}$. Считаем, не теряя общности, $y_{0}>1$. Если $z(a+\xi) \leqslant 2 k a$, то найдем $x_{0}$ (а затем $\left.y_{0}\right)$ из условия $z\left(x_{0}\right)=y_{1}, y_{1}=2 k a$; тогда $a\left(2 k+c y_{0}^{\nu-1}\right) \geqslant c_{1} y_{0}^{2 \nu-1}$, $a \geqslant c_{2} y_{0}^{\nu}$. Если же $z(a+\xi)>2 k a$, то принимаем $y_{0}=k(a+\xi)^{\theta}, y_{1}=z(a+\xi)$. Так или иначе, $1<y_{0}<y_{1}, y_{0} \leqslant c_{3} a^{\theta}$ и $y_{1} \geqslant 2 k a$. Простые геометрические доводы, учитывающие вогнутость функции $y=k x^{\theta}$, показьвают, что расстояние от любой точки $\lambda=a+i y, y_{0} \leqslant y \leqslant y_{1}$, до ветви параболы с $x \geqslant a+\xi$ превосходит величину $\rho(y)=\left(\xi^{2}+\left(y-y_{0}\right)^{2}\right)^{1 / 2}$. Тем более это верно при $0 \leqslant x<a-\xi$.

Полагаем $\chi(y)=\xi$ на $\left[0, y_{0}\right], \chi(y)=\rho(y)$ на $\left(y_{0}, y_{1}\right], \chi(y)=(y-k a) /\left(1+k^{2}\right)^{1 / 2}$ при $y>y_{1}$ и $\chi(y)=\chi(-y)$ при $y<0$. Как видим, $\chi(y) \leqslant r(a+i y)$ при $y_{0}<y \leqslant y_{1}$. На $\left[0, y_{0}\right]$ подобная оценка очевидна, а для $y>y_{1}$ установлена ранее.

$\mathrm{C}$ другой стороны, $\chi(y) \leqslant|a+i y|$ на $\mathbb{R}$. Имеем также $|a+i y|^{\alpha} \leqslant a^{\alpha}+y_{0}^{\alpha}$ при $0 \leqslant y \leqslant y_{0},|a+i y|^{\alpha} \leqslant a^{\alpha}+y_{0}^{\alpha}+\left(y-y_{0}\right)^{\alpha}$ при $y_{0}<y \leqslant y_{1}$ и $|a+i y|^{\alpha} \leqslant a^{\alpha}+$ $(k a)^{\alpha}+(y-k a)^{\alpha}$ при $y>y_{1}$. Оценивая теперь правую часть $(11)$, приходим к неравенству

$$
m(a) \leqslant c_{4} a^{\alpha} / \xi+c_{5} a^{\alpha+\theta} / \xi^{2}
$$

Во всех трех случаях мы использовали декларированную выше свободу выбора параметров $a, \xi$.

Итак, условие на $\xi$ в теореме 2.3 при достаточно больших $a>0$ обретает такую форму: $\xi \geqslant c a^{\alpha / 2}$ для $0 \leqslant \theta \leqslant \alpha / 2$ и $\xi \geqslant c a^{(\alpha+\theta) / 3}$ для $\alpha / 2<\theta \leqslant 1$, где $c=$ $c\left(A, F, T_{0}\right)$. Пороговое значение $\theta=\alpha / 2$ возникает из равенства $(\alpha+\theta) / 3=\alpha / 2$.

Получаем, тем самым, важное следствие теоремы 2.3. 
Теорема 2.8. Пусть пространство X гильбертово, оператор А обладает свойством (3) и выполнено предположение а) теоремы 2.3. Допустим еще, что множество $\mathscr{R}$ содержит полосы $\Gamma\left(a_{n}, \xi_{n}\right)$ с $a_{n}, \xi_{n} \rightarrow \infty$ при $n \rightarrow \infty u$ $\Sigma \subset \Omega(k, \theta) \cap \Omega(k, 1)$ для некоторых $k>0, \theta \in[0,1]$. Возьмем $\beta=\alpha / 2$ при $0 \leqslant \theta \leqslant \alpha / 2$ u $\beta=(\alpha+\theta) / 3$ для $\alpha / 2<\theta \leqslant 1$.

Тогда если $a_{n}^{\beta}=o\left(\xi_{n}\right)$ при $n \rightarrow \infty$, то динамика уравнения (1) на аттракторе конечномерна и справедливо соотношение (7) с подходящим $a>0$.

Отметим, что $\beta \leqslant(\alpha+1) / 3<2 / 3$. Кроме того, $\theta=0$, коль скоро $\Sigma \subset \mathbb{R}$.

Если $A$ - самосопряженный положительный оператор в гильбертовом пространстве $X$, то нетрудно установить (варьируя параметр $\omega$ ) включение $\Sigma \subset \Omega(k, \alpha)$ с надлежашим $k>0$, так что в теореме 2.8 имеем $a_{n}^{2 \alpha / 3}=o\left(\xi_{n}\right)$ при $n \rightarrow \infty$. Известное же условие спектрального скачка (см. [12], [10], [13]), гарантируюшее существование инерциального многообразия уравнения (1), дает здесь в сопоставимых терминах более жесткое (для значений $\alpha>0$ ) ограничение $a_{n}^{\alpha}=o\left(\xi_{n}\right)$ при $n \rightarrow \infty$. Таким образом, в классе эволюционных систем (1) конечномерность предельной динамики выглядит свойством более общего порядка, чем наличие инерциального многообразия.

ЗАмечАниЕ 2.9. Предположение а) теоремы 2.3 относительно поля операторов $B(u, v)$ можно ослабить, заменив требование $\mathscr{L}\left(X^{\alpha}\right)$-ограниченности корректирующего поля $T_{0}$ в $(4 \mathrm{a})$ на $\mathscr{L}\left(X^{\alpha}, X^{\alpha-\varepsilon}\right)$-ограниченность с достаточно малым $\varepsilon>0$. Соответствующая версия теоремы 2.8 должна привести к новым приложениям развитой здесь и в [1] теории.

\section{§3. Уравнение в частных производных}

Покажем, что полученные результаты позволяют установить конечномерность предельной динамики уравнений (2). Дифференциальньй оператор $\partial_{x x} h=h_{x x}$ в $L^{2}(0,1)$ рассматриваем либо с краевыми условиями Штурма

$$
h(0) \cos \mu_{0}+h_{x}(0) \sin \mu_{0}=0, \quad h(1) \cos \mu_{1}+h_{x}(1) \sin \mu_{1}=0
$$

при $\mu_{0}, \mu_{1} \in(-\pi / 2, \pi / 2]$, либо с периодическими условиями

$$
h(0)=h(1), \quad h_{x}(0)=h_{x}(1) .
$$

Необходимые для дальнейшего изложения сведения о пространствах дифференшируемых функций можно найти в [22]-[24].

Пусть $\mathfrak{I}=[0,1]$ в случае (12а) и $\mathfrak{I}-$ окружность длины 1 в случае (12б). Обозначаем через $\mathscr{H}^{s}=\mathscr{H}^{s}(\mathfrak{I})$ обобшенные $L^{2}$-пространства Соболева с произвольными $s \geqslant 0$. Заметим, что при $s>1 / 2$ пространство $\mathscr{H}^{s}$ суть банахова алгебра $[22$, п. 2.8.3]. Оператор $u \rightarrow u_{x}$ непрерывен из $\mathscr{H}^{s+1}$ в $\mathscr{H}^{s}$. Используя теоремы вложения, нетрудно заключить, что для целых $s, \nu \geqslant 1$ и гладкой функции $g: \Im \times \mathbb{R}^{2} \rightarrow \mathbb{R}$ отображение $u \rightarrow g\left(x, u, u_{x}\right)$ принадлежит классу $B C^{\nu}\left(\mathscr{H}^{s+1}, \mathscr{H}^{s}\right)$ при $g \in C^{s+\nu}$ и классу ${ }^{4} B C\left(\mathscr{H}^{s+1}, \mathscr{H}^{s}\right)$ при $g \in C^{s}$.

Требования к функции $f(x, u, p): \mathfrak{I} \times \mathbb{R}^{2} \rightarrow \mathbb{R}$ в (2) выглядит так.

\footnotetext{
${ }^{4}$ Классы отображений $B C^{\nu}, B C$ определены в $\S 1$.
} 
ПРЕДПОЛОЖЕНИЕ 3.1. Функиия $f$ принадлежит $C^{3}$. Если в случае (12а) $\mu_{j}=0$ для $j=0$ или $j=1$, mо $f(j, 0, p)=0$ для всех $p \in \mathbb{R}$.

Сведем уравнение (2) к виду (1) с $X=L^{2}(\mathfrak{I})$. Линейный оператор $\partial_{x x}$ самосопряжен в $X$. При надлежашем выборе числа $\varkappa \geqslant 0$ оператор $A=\varkappa I-\partial_{x x}$ положительно определен $[25$, гл. 1] и дискретен, удовлетворяет, тем самым, гипотезе (H1) и порождает гильбертову полушкалу $\left\{X^{\alpha}\right\}_{\alpha \geqslant 0}$. Как известно $\left[23\right.$, гл. 5], $X^{\alpha}-$ замкнутые подпространства (с эквивалентной нормой) в $\mathscr{H}^{2 \alpha}$, причем $X^{\alpha}=\mathscr{H}^{2 \alpha}$ в случае (12б). Последнее верно и в ситуации (12а) для $\alpha \leqslant 1 / 4$ (для $\alpha \leqslant 3 / 4$, когда $\left.\mu_{0}, \mu_{1} \neq 0\right)$. При $\alpha>3 / 4$ имеют место непрерывные вложения $X^{\alpha} \subset C^{1}(\mathfrak{I})$, $X^{\alpha+1 / 2} \subset C^{2}(\mathfrak{I})$. Непрерьвно, конечно, и вложение $C(\mathfrak{I}) \subset X$. Отсюда, в частности, делаем вывод, что $F \in B C^{3}\left(X^{\alpha}, X\right)$ для отображения $F: u \rightarrow \varkappa u+f\left(x, u, u_{x}\right)$. Если при каком-то $\alpha \in(3 / 4,1)$ уравнение $(2)$ диссипативно в $X^{\alpha}$, то для его абстрактной формы $\partial_{t} u=-A u+F(u)$ справедливы основные гипотезы (Н1)-(Н3), а стало быть, и все конструкции $\S 1,2$. При этом фактически избыточное по сравнению c (Н2) предположение 3.1 о нелинейной части (2) влечет дополнительные качества фазовой динамики. Пусть $\mathscr{A}$ - аттрактор и $\left\{\Phi_{t}\right\}-$ диссипативньй полупоток (2) в $X^{\alpha}, \mathscr{N}=\mathscr{A} \times \mathscr{A}, Y$-пространство Банаха.

ЗАмЕчАниЕ 3.2 . В предположениях 3.1 и (Н3) с $\alpha \in(3 / 4,1)$ уравнение (2) обладает следующими свойствами:

а) аттрактор $\mathscr{A}$ ограничен в $X^{3 / 2}$ или в $X^{2}$ при условиях (12а) или (12б) соответственно;

б) регулярно всякое непрерьвное в $\left(X^{\alpha} \times X^{\alpha}\right)$-метрике поле П: $\mathscr{N} \rightarrow Y$, продолжимое до $C^{1}$-отображения $X^{1} \times X^{1} \rightarrow Y$.

В самом деле, принимая во внимание связь между $X^{s}$ и $\mathscr{H}^{2 s}$, находим из 3.1, что $F \in B C^{2}\left(X^{1}, X^{1 / 2}\right)$, а в случае $(12 б)$, сверх того, $F \in B C^{1}\left(X^{3 / 2}, X^{1}\right)$. Замечания $1.2,1.3$ позволяют теперь установить компактность $\mathscr{A}$ в $X^{1}$ и гладкость отображения $(t, u) \rightarrow \Phi_{t} u:(0, \infty) \times X^{1} \rightarrow X^{1}$, что обеспечивает регулярность (см. определение 2.1) тождественного вложения $\mathscr{N} \rightarrow X^{1} \times X^{1}$, а значит, и свойство б). Наконец, утверждения а) следуют из указанных замечаний непосредственно.

Различные признаки $X^{\alpha}$-диссипативности (2) можно получать на основе известных априорных оценок [26], [9], [27] решений таких уравнений с помощью абстрактных функционально-аналитических методов [2], [9], [28]. Например, рассмотренные в $[9$, гл. $1, \S 7]$ ограничения $f(x, u, 0) \operatorname{sign} u \rightarrow-\infty$ при $|u| \rightarrow \infty$ равномерно по $x \in \mathfrak{I}$,

$$
|f|+\left|f_{x}\right|+\left|f_{u}\right| \leqslant M(u)\left(1+p^{2}\right), \quad\left|f_{p}\right| \leqslant M(u)(1+|p|),
$$

на удовлетворяющую предположению 3.1 функцию $f(x, u, p)$ гарантируют $X^{\alpha}$-диссипативность (при $3 / 4<\alpha<1$ ) задач (2), (12a) с $\mu_{0}=\mu_{1}=0$ и периодической задачи (2), (12б). Действительно, при каждом $u_{0} \in X^{\alpha} \subset C^{1}(\mathfrak{I})$ уравнение (2) имеет локальное решение $u(t) \in C\left(\left[0, t^{*}\right), X^{\alpha}\right), u(t) \in X^{1}$ на $\left(0, t^{*}\right)$. Если же $u_{0} \in X^{1}$, то в силу замечания $1.2 u(t) \in C\left(\left[0, t^{*}\right), X^{1}\right)$. В любом случае $u(t) \in X^{3 / 2}$ для $t \in\left(0, t^{*}\right)$, т.е. $u(t)$ принадлежит классу Гёльдера $C^{2+\delta}(\mathfrak{I})$ с некоторым $0<\delta<1$, и применимы теоремы 1.7.2, 2.5.1 из [9]. Хотя цитированные утверждения подразумевают граничное условие Дирихле, все необходимые для их 
вывода рассуждения проходят и в периодической ситуации. Так или иначе, для уравнений (2) устанавливается глобальное по $t>0$ существование решений

$$
u(t)=\Phi_{t} u_{0} \in X^{\alpha} \cap C^{2+\delta}(\mathfrak{I}), \quad u_{0} \in X^{\alpha},
$$

а также равномерная $(C(\mathfrak{I}), C(\mathfrak{I}))$-ограниченность по $t \geqslant 0$ фазового полупотока $\left\{\Phi_{t}\right\}$ в $X^{\alpha}$ и наличие инвариантного компакта $\mathscr{A} \subset X^{\alpha}$, являющегося $(C(\mathfrak{I})$, $\left.C^{2+\delta}(\mathfrak{I})\right)$-аттрактором. Здесь сохранена удобная терминология [9]. Далее, вложения $X^{\alpha} \subset C(\mathfrak{I})$ и $C^{2+\delta}(\mathfrak{I}) \subset \mathscr{H}^{2}$ непрерывны, значит, $\mathscr{A}$ есть $\left(X^{\alpha}, X^{1}\right)$-аттрактор $\left(\mathscr{A}\right.$ равномерно притягивает шары $\mathscr{B} \subset X^{\alpha}$ при $t \rightarrow+\infty$ по норме $\left.X^{1}\right)$, откуда с запасом следует диссипативность (2) в $X^{\alpha}$.

Поясним, почему для задач $(2),(12)$ не удается построить инерциальное многообразие. Если занумеровать собственные числа $\lambda_{n}$ оператора $A$ по возрастанию и положить $a_{n}=\left(\lambda_{n+1}+\lambda_{n}\right) / 2, \xi_{n}=\left(\lambda_{n+1}-\lambda_{n}\right) / 2$ для $n \geqslant 1$, то [25] $a_{n} \sim c n^{2}$ и $\xi_{n} \sim$ cn при $n \rightarrow \infty, c=$ const. Условие спектрального скачка $a_{n}^{\alpha}=o\left(\xi_{n}\right)$, обеспечивающее "асимптотическую конечномерность” фазовой динамики, подразумевало бы тут неравенство $\alpha<1 / 2$, принципиально невозможное даже в самых жестких ограничениях на зависящую от $u_{x}$ нелинейность $f$.

Между тем, предельная динамика уравнения (2) конечномерна.

Теорема 3.3. Допустим, функиия $f: \mathfrak{I} \times \mathbb{R}^{2} \rightarrow \mathbb{R}$ удовлетворяет предположению 3.1 и $\alpha \in(3 / 4,1)$. Тогда если уравнение (2) с каким-то из краевых условий (12) диссипативно в $X^{\alpha}$, то его фазовая динамика на аттракторе конечномерна.

ДокАЗАТЕЛЬСтво сводится к проверке требований теоремы 2.8. Соотношение (3), разумеется, выполнено для положительно определенного оператора $A$. Если $u, h \in X^{\alpha}$, то

$$
D F(u) h=\varkappa h+f_{u}\left(x, u, u_{x}\right) h+f_{p}\left(x, u, u_{x}\right) h_{x}
$$

Полагаем

$$
b(x ; u, v)=\int_{0}^{1} f_{p}\left(x, w, w_{x}\right) d \tau, \quad b_{0}(x ; u, v)=\int_{0}^{1} f_{u}\left(x, w, w_{x}\right) d \tau
$$

при $u, v \in X^{\alpha}, w=\tau u+(1-\tau) v$. Данные выражения удобно трактовать как интегралы Бохнера со значениями в тех или иных функциональных пространствах, что позволяет применять к их анализу соответствующую технику [29, гл. 3]. Так как $f \in C^{3}$, то $f_{p}, f_{u} \in C^{2}$ и отображения $\Pi_{1}: u \rightarrow f_{p}\left(x, u, u_{x}\right), \Pi_{2}: u \rightarrow f_{u}\left(x, u, u_{x}\right)$ принадлежат классу $B C\left(\mathscr{H}^{3}, \mathscr{H}^{2}\right)$. Согласно замечанию 3.2 , а) выпуклая оболочка $\mathscr{A}^{c}$ аттрактора $\mathscr{A}$ ограничена в $X^{3 / 2} \subset \mathscr{H}^{3}$, следовательно, множества $\Pi_{1} \mathscr{A}^{c}, \Pi_{2} \mathscr{A}^{c}$ ограничены в $\mathscr{H}^{2}$. Это влечет равномерную по $(u, v) \in \mathscr{N}$ ограниченность функций $b, b_{0}\left(\right.$ а также $\left.b^{2}\right)$ в нормах банаховых алгебр $\mathscr{H}^{2}$ и $C^{1}(\mathfrak{I})$. В случае $(12 б)$, исходя из $X^{2}$-ограниченности $\mathscr{A}$, получаем таким же образом равномерную $\mathscr{H}^{3}$-ограниченность указанных функций.

Запишем, следуя формату (4),

$$
\begin{gathered}
T(u, v) h=T_{0}(u, v) h+\varkappa h+b_{0}(x ; u, v) h+b(x ; u, v) h_{x} \\
B(u, v)=(\omega+\varkappa) I-\partial_{x x}-T(u, v)
\end{gathered}
$$


для $u, v \in \mathscr{A}$. Напомним, что поле операторов $T_{0}$ на $\mathscr{N}$ должно быть ограничено со значениями в $\mathscr{L}\left(X^{\alpha}\right)$ и регулярно со значениями в $\mathscr{L}\left(X^{\alpha}, X\right)$. Возьмем сперва $T_{0}=0$ и выберем число $\omega>0$ с помощью леммы 4.1. Обозначим через $S(u, v)$ непрерывный в $X=L^{2}(\mathfrak{I})$ оператор умножения на положительную функцию $\psi(x ; u, v) \in C^{2}[0,1]$ такую, что $(\ln \psi)_{x}=b / 2$ и $\left.\psi\right|_{x=0}=1$. В периодическом случае, вообше говоря, $\left.\psi\right|_{x=1} \neq 1$ и $\psi \notin C(\mathfrak{I})$. Преобразование $\eta=S(u, v) h$ для $h \in X^{1}$ дает возможность представить операторы $B=B(u, v)$ в виде $B=S^{-1} H S$, где $H=H(u, v)$,

$$
H(u, v) \eta=\omega \eta-\eta_{x x}-q(x ; u, v) \eta
$$

и $q=b_{0}-b^{2} / 4-b_{x} / 2$. Функции $q \in \mathscr{H}^{1} \subset C(\mathfrak{I})$ равномерно по $u, v \in \mathscr{A}$ ограничены в норме $\mathscr{H}^{1}$. Для операторов $H$ область определения $\mathscr{D}(H)$ совпадает с $S \mathscr{D}(B)$, где $\mathscr{D}(B)=\mathscr{D}(A)=X^{1}$, т.е. при переходе от $B$ к $H$ краевые условия могут измениться. Тем не менее, тип (12a) в целом сохраняется и операторы $H(u, v)$ оказываются самосопряженными в $X$. Условия же (12б) трансформируются в $\eta(1)=\rho \eta(0)$, $\eta_{x}(1)=\rho \eta_{x}(0)$ с $\rho=\left.\psi\right|_{x=1}$ (ибо функции $f_{p}, b$ периодичны по $x$ ), и тут $H(u, v)$ при $\rho \neq 1$ не являются даже нормальными. В обоих случаях ${ }^{5}\|S\|=|\psi|_{C}$, регулярность поля операторов $S: \mathcal{N} \rightarrow \mathscr{L}(X)$ будет следовать из регулярности поля функций $\psi(\cdot ; u, v): \mathscr{N} \rightarrow C[0,1]$, а последняя, в свою очередь, из регулярности поля функций $b(\cdot ; u, v): \mathscr{N} \rightarrow C(\mathfrak{I})$. Пользуясь $C^{1}$-гладкостью нелинейного оператора $(u, v) \rightarrow f_{p}\left(x, w, w_{x}\right): X^{\alpha} \times X^{\alpha} \rightarrow C(\mathfrak{I})$ с $w=\tau u+(1-\tau) v, \tau \in[0,1]$ и дифференцируя под знаком интеграла в (13) по параметру $(u, v) \in X^{\alpha} \times X^{\alpha}$, заключаем, что отображение $\Pi:(u, v) \rightarrow b(\cdot ; u, v)$ принадлежит классу $C^{1}\left(X^{\alpha} \times X^{\alpha}, C(\mathfrak{I})\right)$, а значит, его сужение на $\mathscr{N}$ регулярно. Точно так же доказывается регулярность поля функций $b_{0}: \mathcal{N} \rightarrow C(\mathfrak{I})$ в (13), а регулярность поля $b^{2}: \mathscr{N} \rightarrow C(\mathfrak{I})$ явствует из регулярности $b$ и мультипликативной структуры $C(\mathfrak{I})$.

Итак, в случае Штурма (12а) операторы $H(u, v)$ самосопряжены, поле $S$ регулярно и $\left\|S^{-1}\right\|=\left|\psi^{-1}\right|_{C} \leqslant$ const на $\mathscr{N}$, следовательно, $B: \mathscr{N} \rightarrow \mathscr{L}\left(X^{1}, X\right)$ есть поле операторов равномерно скалярного типа в смысле определения 2.2. Так как совокупный спектр $\Sigma=\Sigma(B) \subset \mathbb{R}^{+}$, то условие $\Sigma \subset \Omega(k, \theta) \cap \Omega(k, 1)$ теоремы 2.8 вьполняется тривиально с $\theta=0$ и любым $k>0$.

Установим необходимую разреженность $\Sigma$. Пользуясь асимптотикой $[25$, гл. 1$]$ собственных чисел операторов вида (15), обнаруживаем, что в терминах теоремы 2.8 здесь можно взять $a_{n}=\pi^{2} n^{2}+c n, \xi_{n}=\pi^{2} n+c_{1} \mathrm{c} n \geqslant n_{0}$, где величины $c$, $c_{1}, n_{0}$ зависят от $\mu_{0}, \mu_{1}$ и общей мажоранты $\mathscr{H}^{1}$-норм функций $q(\cdot ; u, v)$. В этих же терминах $\beta=\alpha / 2<1 / 2, a_{n}^{\beta}=o\left(\xi_{n}\right)$ при $n \rightarrow \infty$ и для задач (2), (12a) теорема 3.3 доказана.

Обратимся к периодическому случаю (12б). Положим теперь $T_{0}(u, v) h=$ $-q(x ; u, v) h$ в равенстве (14a). Соответственно изменится поле операторов $B(u, v)$ в (14б). В данной ситуации пространство $X^{\alpha}=\mathscr{H}^{2 \alpha}$ - банахова алгебра и, как уже говорилось, функции $b, b_{0}, b^{2}$ равномерно по $(u, v) \in \mathscr{N}$ ограничены в норме $\mathscr{H}^{3}$, а потому $|q(\cdot ; u, v)|_{1} \leqslant$ const. Тем более, $|q(\cdot ; u, v)|_{\alpha} \leqslant$ const, так что мультипликаторы $T_{0}(u, v)$ принадлежат $\mathscr{L}\left(X^{\alpha}\right)$ и $\left\|T_{0}(u, v)\right\|_{\alpha} \leqslant$ const для $u, v \in \mathscr{A}$. Из (14б), (15) следует представление $B=S^{-1} H_{0} S$, в котором $S(u, v)$ - операторы, определенные вьше, а $H_{0}=H_{0}(u, v)=\omega I-\partial_{x x}$ с краевыми условиями

\footnotetext{
${ }^{5}|\cdot|_{C}-$ норма в $C[0,1]$.
} 
$h(1)=\rho h(0), h_{x}(1)=\rho h_{x}(0)$ и $\rho=\rho(u, v)=\left.\psi(x ; u, v)\right|_{x=1}>0$. При этом собственные числа $\lambda$ и собственные функции $\chi(x)$ оператора $\left(-\bar{\partial}_{x x}\right)$ легко вычисляются:

$$
\begin{array}{lll}
\lambda_{0}=-\ln ^{2} p, & \lambda_{n, 1}=(2 \pi n-i \ln \rho)^{2}, & \lambda_{n, 2}=(2 \pi n+i \ln \rho)^{2}, \\
\chi_{0}=\rho^{x}, & \chi_{n, 1}=\rho^{x} e^{2 \pi n i x}, & \chi_{n, 2}=\rho^{x} e^{-2 \pi n i x}
\end{array}
$$

для $n \geqslant 1$. Система функций $\left\{\chi_{0}, \chi_{n, 1}, \chi_{n, 2}\right\}$ полна и ортогональна в $L^{2}(\mathfrak{I})$ с весом $\rho^{-2 x}$, значит, $H_{0}=S_{0}^{-1} H_{1} S_{0}$, где операторы $H_{1}=H_{1}(u, v)$ нормальны в $X$, $\mathscr{D}\left(H_{1}\right)=\mathscr{D}(A)=X^{1}, S_{0}(u, v) h=\rho^{-x} h$ для $h \in X$. Как видим, $B=S_{1}^{-1} H_{1} S_{1} \mathrm{c}$ $S_{1}=S_{0} S$, причем $\left\|S_{1}^{-1}\right\| \leqslant\left\|S^{-1}\right\| \cdot\left\|S_{0}^{-1}\right\| \leqslant$ const на $\mathscr{N}$.

В контексте требований теоремы 2.8 и определения 2.2 нам нужна регулярность полей операторов $S_{1}: \mathscr{N} \rightarrow \mathscr{L}(X), T_{0}: \mathscr{N} \rightarrow \mathscr{L}\left(X^{\alpha}, X\right)$. Доказанная выше регулярность полей $\psi, S$ влечет то же свойство для полей $S_{0}, S_{1}: \mathscr{N} \rightarrow \mathscr{L}(X)$. Вложение $X^{\alpha} \subset C(\mathfrak{I})$ непрерывно, так что $\left\|T_{0}\right\|_{\alpha, 0} \leqslant c|q|$ (тут $|\cdot|$ - норма в $X$, постоянная $c$ не зависит от $q$ ) и поле $T_{0}$ регулярно, коли таковым является поле функций $q: \mathcal{N} \rightarrow X, q=b_{0}-b^{2} / 4-b_{x} / 2$. Поля функций $b_{0}, b^{2}$ на $\mathscr{N}$ регулярны со значениями в $C(\mathfrak{I})$ и, тем более, со значениями в $X$.

Пусть $\Pi_{\tau}(u, v)=\left(f_{p}\left(x, w, w_{x}\right)\right)_{x} \mathrm{c} w=\tau u+(1-\tau) v, \tau \in[0,1]$ и произвольными $u=u(x), v=v(x) \in X^{1}$, a $(u, v)$ - результат интегрирования $\Pi_{\tau}(u, v)$ по $\tau$. Ясно, что $\Pi(u, v)=(b(x ; u, v))_{x}$ для $u, v \in \mathscr{A} \subset X^{1}$, поэтому в силу замечания 3.2, б) регулярность поля функций $b_{x}: \mathcal{N} \rightarrow X$ будет следствием включений $\left.\Pi\right|_{\mathcal{N}} \in$ $C(\mathscr{N}, X), \Pi \in C^{1}\left(X^{1} \times X^{1}, X\right)$. Поскольку $f \in C^{3}$ и $f_{p} \in C^{2}$, то отображение $u \rightarrow f_{p}\left(x, u, u_{x}\right)$ принадлежит классу $B C^{1}\left(X^{1}, X^{1 / 2}\right)$, стало быть, $\Pi_{\tau} \in C^{1}\left(X^{1} \times\right.$ $\left.X^{1}, X\right)$. Дифференцируя интегральное выражение для П по параметру $(u, v) \in$ $X^{1} \times X^{1}$, находим, что $\Pi \in C^{1}\left(X^{1} \times X^{1}, X\right)$. Далее, операторы $u \rightarrow g\left(x, u, u_{x}\right)$, $g=f_{p x}, f_{p u}, f_{p p}$, действуют непрерьвно из $X^{\alpha}$ в $C(\Im)$ и $\left(f_{p}\left(x, u, u_{x}\right)\right)_{x}=f_{p x}+$ $f_{p u} u_{x}+f_{p p} u_{x x}$ для $u \in \mathscr{A}^{c} \subset X^{1}$. По лемме 1.1 функция $u \rightarrow A u: \mathscr{A} \rightarrow X$, $A u=\varkappa u-u_{x x}$, непрерьвна в $X^{\alpha}$-метрике. Последнее верно и для отображений $u \rightarrow u_{x x}, u \rightarrow u_{x}$ множества $\mathscr{A}^{c}$ в $X$. Таким образом, $\Pi_{\tau},\left.\Pi\right|_{\mathscr{N}} \in C(\mathscr{N}, X)$, поля $b_{x}, q, T_{0}$ регулярны и $B(u, v)$ - поле операторов равномерно скалярного типа на $\mathscr{N}$.

Остается уточнить значение $\omega$ в (14б) и найти достаточно широкие лакуны во множестве $\Sigma=\Sigma(B) \subset \mathbb{C}=\{x+i y\}$. Пусть $\omega>\ln ^{2} \rho(u, v)+1$ на $\mathscr{N} ;$ тогда из (16) следует, что $\Sigma \subset \Omega(k, \theta), \theta=1 / 2, k=2(\omega-1)^{1 / 2}$. Фактически же $\Sigma$ принадлежит области $|y| \leqslant k(x-1)^{\theta}$, а значит, $\Sigma \subset \Omega\left(k_{1}, 1\right)$ с $k_{1}=k_{1}(\omega)>0$. При этом в формате теоремы 2.8 естественно взять $a_{n}=4 \pi^{2}\left(n^{2}+n+1 / 2\right), \xi_{n}=2 \pi^{2}(n+1 / 2)$ для $n>\omega / 2 \pi^{2}$. Кроме того, $\theta>\alpha / 2$ и $\beta=(\alpha+\theta) / 3<1 / 2$, ибо $\alpha<1$, а потому $a_{n}^{\beta}=o\left(\xi_{n}\right)$ при $n \rightarrow \infty$. Как результат получаем конечномерность предельной фазовой динамики для задачи (2), (12б). Теорема 3.3 доказана полностью.

Отметим, что теорема 2.8 обеспечивает еше свойство (7) динамики (2) на аттракторе $\mathscr{A}$, причем структура числовой формы $W_{a}$ и значение параметра $a$ в $(7)$ зависят от выбора $T_{0}, \omega$ в (14).

Конечномерной предельной динамикой обладает, например, уравнение реакции-диффузии с нелинейной конвекцией

$$
u_{t}=u_{x x}+(g(x, u))_{x}+g_{0}(x, u), \quad x \in(0,1),
$$

при стандартных условиях в $x=0,1$. Ограничения на гладкие функции $g, g_{0}$ диктуются предположением 3.1 и требованием диссипативности данного уравнения в $X^{\alpha} \mathrm{c} \alpha \in(3 / 4,1)$. 
ЗАмечание 3.4. Теорема 3.3 обобщается на одномерные системы

$$
u_{t}^{j}=\left(d(x) u_{x}^{j}\right)_{x}+f_{j}\left(x, u, u_{x}\right), \quad 1 \leqslant j \leqslant l,
$$

с $u=\left(u^{1}, u^{2}, \ldots, u^{l}\right)$, граничным условием Дирихле и гладким на $[0,1]$ коэффициентом $d(x)>0$, если использовать в доказательстве упоминавшийся уже аналог преобразования Лиувилля [16].

По-видимому, конечномерность предельной динамики может быть доказана также для полулинейных параболических уравнений на $(0,1)$ порядка выше двух при не слишком патологических граничных условиях и подходяших ограничениях на нелинейную часть. Это предполагает, однако, определенную модификацию построений $\S 2$ (см. замечание 2.9$)$ и настояшего параграфа.

\section{§4. Приложение}

Здесь собраны технического рода утверждения, связанные со свойствами производной векторного поля $F(u)-A u$ уравнения (1) в банаховом пространстве $X$. Как и ранее, исходим из основных гипотез (H1)-(Н3). Записываем $\lambda=x+i y$ для $\lambda \in \mathbb{C}$. Напоминаем, что $\mathscr{A}$ - аттрактор (1) в $X^{\alpha}, \mathscr{N}=\mathscr{A} \times \mathscr{A}$, спектр $\sigma(A)$ принадлежит сектору $\Omega=\{\lambda:|y|<k x\}$ с $k>0$ и $\|R(\lambda ; A)\| \leqslant M /(1+|\lambda|)$ в $\Omega_{1}=\mathbb{C} \backslash \Omega$ для резольвенты $R(\lambda ; A)=(A-\lambda I)^{-1}$. Обозначения пространств и норм линейных операторов соответствуют обозначением, принятым в $\S 2$. Поля операторов $T, B$ на $\mathscr{N}$ задаются равенствами (4а), (4б) с $\omega \geqslant \omega_{0}$, где $\omega_{0}$ - постоянная из нижеследующей леммы, причем поле $T: \mathcal{N} \rightarrow \mathscr{L}\left(X^{\alpha}, X\right)$ регулярно в смысле определения 2.1 .

Лемма 4.1. Для $\omega \geqslant \omega_{0}>0 u B=B(u, v)$ c $u, v \in \mathscr{A}$ спектр $\sigma(B) \subset \Omega$,

$$
\|R(\lambda ; B)\| \leqslant \frac{M_{1}}{1+|\lambda|}
$$

при $\lambda \in \Omega_{1} u$

$$
\left\|A^{\alpha} B^{-\alpha}\right\| \leqslant L, \quad\left\|B^{\alpha} A^{-\alpha}\right\| \leqslant L .
$$

Константы $M_{1}, L$ зависят лишь от $A$. Постоянная $\omega_{0}$ зависит от $A, F$, $T_{0}$, где $T_{0}-$ поле операторов в (4a).

Будем использовать неравенство моментов [2, теорема 1.4.4]

$$
\left\|A^{\alpha} V\right\| \leqslant \Theta\|A V\|^{\alpha}\|V\|^{1-\alpha}
$$

для секториального оператора $A$ с $V, A V \in \mathscr{L}(X)$ и $\Theta=\Theta(k, M)$. Всюду дальше $c, c_{1}, \ldots$ - константы, зависящие только от $A, F, T_{0}$. Условимся отождествлять зависимость тех или иных величин от оператора $A$ и от его параметров $(k, M)$, хотя последние и выбираются по $A$ неоднозначно. 
ДокАЗАТЕЛЬСТво. Регулярность поля операторов $T=T(u, v)$ влечет соотношение $\left\|T A^{-\alpha}\right\| \leqslant c$ на $\mathscr{N}$. Пусть $R=R(\lambda ; A)$ для $\lambda \in \Omega_{1}$; тогда (формально) $R(\lambda ; A-T)=R(I-T R)^{-1}$ и $\lambda \notin \sigma(A-T)$, если $\|T R\|<1$. Ясно, что

$$
\|T R\|=\left\|T A^{-\alpha} A^{\alpha} R\right\| \leqslant c\left\|A^{\alpha} R\right\| .
$$

Так как $A R=I+\lambda R \in \mathscr{L}(X)$, то (19) обеспечивает неравенство

$$
\left\|A^{\alpha} R\right\| \leqslant \Theta\|A R\|^{\alpha}\|R\|^{1-\alpha} .
$$

Отсюда явствует, что

$$
\|T R\| \leqslant c \Theta\left(\|R\|^{1-\alpha}+|\lambda|^{\alpha}\|R\|\right) .
$$

Используя оценку на $R(\lambda ; A)$, получаем

$$
\|T R\| \leqslant c_{1}(1+|\lambda|)^{\alpha-1}, \quad c_{1}=c \Theta\left(M^{1-\alpha}+M\right) .
$$

Таким образом, $\|T R\| \leqslant 1 / 2$ при $|\lambda|^{1-\alpha} \geqslant 2 c_{1}$ и геометрические соображения показывают, что для всех $u, v \in \mathscr{A}$ спектр $\sigma(A-T(u, v))$ лежит в секторе $|y|<$ $k\left(x+\omega_{0}\right) \mathrm{c} \omega_{0}=\rho \varkappa, \quad \rho^{1-\alpha}=2 c_{1}, \varkappa=\left(1+k^{-2}\right)^{1 / 2}$, где $\omega_{0}=\omega_{0}(k, M, T)$, т.е. фактически $\omega_{0}$ зависит от $A, F, T_{0}$. Вне указанного сектора $\|T R\| \leqslant 1 / 2$ и $\left\|(I-T R)^{-1}\right\| \leqslant(1-\|T R\|)^{-1} \leqslant 2$, а потому

$$
\|R(\lambda ; A-T)\| \leqslant 2\|R(\lambda ; A)\| \leqslant 2 M /(1+|\lambda|) .
$$

Если теперь $\omega \geqslant \omega_{0}$ в (4б), то $\sigma(B(u, v)) \subset \Omega$ и $\|R(\lambda ; B)\| \leqslant 2 M /(1+|\lambda-\omega|)$ на $\Omega_{1}$. Решив простую задачу на экстремум, находим

$$
|\lambda| \leqslant \varkappa|\lambda-\omega|, \quad\|R(\lambda ; B)\| \leqslant M_{1}(1+|\lambda|)
$$

с $M_{1}=2 \varkappa M$ при $\lambda \in \Omega_{1}$. Ограниченность в $X$ операторов $A^{\alpha} B^{-\alpha}, B^{\alpha} A^{-\alpha}$ вытекает из $[2$, теорема 1.4.6]. Внимательный анализ соответствуюших выкладок [2] показывает, что нормы данных операторов оцениваются через величины $k, M, M_{1}=M_{1}(k, M)$. Это завершает доказательство леммы.

Напомним еше, что $r(\lambda)$ - расстояние от $\lambda \in \mathbb{C}$ до совокупного спектра $\Sigma=$ $\Sigma(B), \mathscr{R}=\mathbb{C} \backslash \Sigma$ и $\Gamma_{a}-$ прямая $x=a$ в $\mathbb{C}$. Как только что установлено, $\Sigma \subset \Omega$, $\mathscr{R} \supset \Omega_{1}$. Проекторы $P_{a}$ определены в $\S 2$.

ЛЕмма 4.2. Если $\Gamma_{a} \subset \mathscr{R}$ при некотором $a>0 u$

$$
\|R(\lambda ; B)\| \leqslant \frac{c}{r(\lambda)} \quad \text { на } \mathscr{R}
$$

для $B=B(u, v), \quad u, v \in \mathscr{A}$, то поле проекторов $P_{a}: \mathscr{N} \rightarrow \mathscr{L}\left(X^{\alpha}\right)$ регулярно, а для $\partial_{t} P_{a}(u, v)$ справедливо интегральное представление (9) с голоморфным по $\lambda \in \mathscr{R}$ и удовлетворяющим оченке $(10)$ ядром $E(\lambda ; u, v) \in \mathscr{L}\left(X^{\alpha}\right)$. 
ДокАЗАТЕЛЬСТВо. Возьмем в $\mathbb{C}$ треугольный положительно ориентированный контур $\Gamma \subset \mathscr{R}$ с вершинами $(0,0),(a,-k a)$ и $(a, k a)$, где $k$ - параметр секториального оператора $A$. Пусть $(u, v) \in \mathscr{N}, T=T(u, v), R=R(\lambda ; B)=R(\lambda ; u, v)$ для $\lambda \in \mathscr{R}$. Исходим из формулы Рисса

$$
P_{a}(u, v)=-\frac{1}{2 \pi i} \int_{\Gamma} R(\lambda ; u, v) d \lambda .
$$

Поскольку $B R=I+\lambda R$, то $B R \in \mathscr{L}(X), B^{\alpha} R \in \mathscr{L}(X)$ и $R \in \mathscr{L}\left(X, X^{\alpha}\right)$. $\mathrm{C}$ учетом (18) имеем

$$
\|R\|_{0, \alpha}=\left\|A^{\alpha} R\right\|=\left\|A^{\alpha} B^{-\alpha} B^{\alpha} R\right\| \leqslant L\left\|B^{\alpha} R\right\| .
$$

Положив $V=R$ в неравенстве (19) и заменив $A$ на секториальный оператор $B$, выводим оценку

$$
\left\|B^{\alpha} R\right\| \leqslant \Theta\left(\|R\|^{1-\alpha}+|\lambda|^{\alpha}\|R\|\right),
$$

причем по лемме $4.1 \Theta=\Theta\left(k, M_{1}\right)=\Theta(A)$.

Как видим из (20),

$$
\|R\|_{0, \alpha} \leqslant c_{1}\left(\|R\|^{1-\alpha}+|\lambda|^{\alpha}\|R\|\right) \leqslant K(\Lambda)
$$

на $\Lambda \times \mathscr{N}$ для произвольного замкнутого множества $\Lambda \subset \mathscr{R}$. Регулярность поля $T: \mathscr{N} \rightarrow \mathscr{L}\left(X^{\alpha}, X\right)$ в сочетании со вторым резольвентным тождеством $[24$, п. 3.2 .1$]$ позволяют установить при каждом $\lambda \in \Lambda$ регулярность поля операторов $R: \mathscr{N} \rightarrow$ $\mathscr{L}\left(X, X^{\alpha}\right)$ наряду с соотношением $\partial_{t} R=R \partial_{t} T R$ и неравенством

$$
\left\|\partial_{t} R\right\|_{0, \alpha} \leqslant c_{2}\left(\|R\|^{1-\alpha}+|\lambda|^{\alpha}\|R\|\right)^{2} \leqslant K_{1}(\Lambda) .
$$

Отметим, что для $\Lambda=(-\infty, 0]$ доводы текущего абзаца верны и без условия $(20)$, если использовать вместо него оценку (17).

Итак, $\|R(\lambda ; u, v)\|_{0, \alpha} \leqslant$ const на $\Gamma \times \mathscr{N}$. Упомянутое резольвентное тождество дает возможность перенести непрерывность поля $R: \mathscr{N} \rightarrow \mathscr{L}\left(X, X^{\alpha}\right)$ с $\lambda \in$ Гна поле $P_{a}: \mathscr{N} \rightarrow \mathscr{L}\left(X, X^{\alpha}\right)$. Функция $\partial_{t} T\left(\Phi_{t} u, \Phi_{t} v\right):(u, v, t) \rightarrow \mathscr{L}\left(X^{\alpha}, X\right)$ ограничена на $\mathscr{N} \times[0, \infty)$ и непрерывна по $t \geqslant 0$, значит, функция $\partial_{t} R\left(\lambda ; \Phi_{t} u, \Phi_{t} v\right):(\lambda, u, v, t) \rightarrow$ $\mathscr{L}\left(X, X^{\alpha}\right)$ ограничена на $\Gamma \times \mathscr{N} \times[0, \infty)$ и непрерывна по $t \geqslant 0$. Тем самым, интеграл (21) можно дифференцировать по параметру $t$ в силу уравнения (1) и поле проекторов $P_{a}$ регулярно со значениями в $\mathscr{L}\left(X, X^{\alpha}\right)$, а тем более со значениями в $\mathscr{L}\left(X^{\alpha}\right)$. Для производной в нуле $\partial_{t} P_{a}(u, v)$ получаем пока выражение типа $(9)$ с голоморфным на $\mathscr{R}$ ядром

$$
E(\lambda)=E(\lambda ; u, v)=-\partial_{t} R(\lambda ; u, v) \in \mathscr{L}\left(X^{\alpha}\right)
$$

и контуром интегрирования $\Gamma$. В силу (18) и тождества $R B^{\alpha}=B^{\alpha} R$ находим, что

$$
\|R\|_{\alpha}=\left\|A^{\alpha} B^{-\alpha} R B^{\alpha} A^{-\alpha}\right\| \leqslant L^{2}\|R\| .
$$

Поскольку $\left\|\partial_{t} T\right\|_{\alpha, 0} \leqslant c_{3}$, то

$$
\|E(\lambda)\|_{\alpha} \leqslant c_{3}\|R\|_{0, \alpha}\|R\|_{\alpha} \leqslant c_{4}\left(\|R\|^{2-\alpha}+|\lambda|^{\alpha}\|R\|^{2}\right)
$$

на $\mathscr{R}$ и искомая оценка (10) следует из неравенства (20). Согласно (17) при $\lambda \rightarrow \infty$ в $\Omega_{1}$ величина $\|R(\lambda ; B)\|=O\left(|\lambda|^{-1}\right)$, а потому $\|E(\lambda)\|_{\alpha}=O\left(|\lambda|^{\alpha-2}\right), \quad \alpha<1$. Отсюда, учитьвая голоморфность $E(\lambda)$, выводим для $\partial_{t} P_{a}(u, v)$ окончательное представление (9) с контуром интегрирования $\Gamma_{a}$. Лемма доказана. 
Лемма 4.3. Пусть $B=B(u, v), \quad u, v \in \mathscr{A} ;$ тогда поля операторов $B^{-\alpha}$ : $\mathscr{N} \rightarrow \mathscr{L}\left(X, X^{\alpha}\right)$ и $B^{\alpha}: \mathscr{N} \rightarrow \mathscr{L}\left(X^{\alpha}, X\right)$ регулярны. Справедлива оченка

$$
\left\|\left(\partial_{t} B^{\alpha}\right) B^{-\alpha}\right\| \leqslant N
$$

на $\mathscr{N}$ с постоянной $N=N\left(A, F, T_{0}\right)$.

ДоказАТЕльСтво. Считаем, не теряя обшности, что $\alpha>0$. Тогда, как известно $[2, \S 1.4]$,

$$
B^{-\alpha}=\frac{\sin \pi \alpha}{\pi} \int_{-\infty}^{0}|\lambda|^{-\alpha} R(\lambda ; B) d \lambda
$$

для $B=B(u, v)$. При $\lambda \leqslant 0$ промежуточные результаты предыдущего доказательства гарантируют регулярность поля $R(\lambda ; B): \mathscr{N} \rightarrow \mathscr{L}\left(X, X^{\alpha}\right)$ и равенство $\partial_{t} R=R \partial_{t} T R$. Найденные там же оценки на $R$ и $\partial_{t} R$ запишем с помошью (17) в виде

$$
\|R\|_{0, \alpha} \leqslant c_{1}(1+|\lambda|)^{\alpha-1}, \quad\left\|\partial_{t} R\right\|_{0, \alpha} \leqslant c_{2}(1+|\lambda|)^{2 \alpha-2} .
$$

Подчеркнем, что все это верно без условия $(20)$ на $R(\lambda ; B)$. Второе резольвентное тождество позволяет перенести непрерывность поля $R: \mathscr{N} \rightarrow \mathscr{L}\left(X, X^{\alpha}\right)$ с $\lambda \leqslant 0$ на поле $B^{-\alpha}: \mathscr{N} \rightarrow \mathscr{L}\left(X, X^{\alpha}\right)$. Функция $\psi(\lambda)=|\lambda|^{-\alpha}(1+|\lambda|)^{2 \alpha-2}$ интегрируема на $(-\infty, 0)$. Применяя теорему Лебега о предельном переходе под знаком интеграла и рассуждая, как выше, заключаем, что выражение (23) можно дифференцировать по параметру $t$ в силу уравнения (1) и поле $B^{-\alpha}$ регулярно. Пользуясь (18), имеем

$$
\left\|B^{\alpha} \partial_{t} R\right\|=\left\|B^{\alpha} A^{-\alpha} A^{\alpha} \partial_{t} R\right\| \leqslant L\left\|\partial_{t} R\right\|_{0, \alpha} \leqslant c_{3}(1+|\lambda|)^{2 \alpha-2} .
$$

Из (23) следует теперь неравенство $\left\|B^{\alpha} \partial_{t} B^{-\alpha}\right\| \leqslant N$ для $u, v \in \mathscr{A}$ с $N=N(A, F$, $\left.T_{0}\right)$.

Осталось заметить, что $B^{\alpha} B^{-\alpha}=I$ и $\left\|B^{\alpha}\right\|_{\alpha, 0}=\left\|B^{\alpha} A^{-\alpha}\right\| \leqslant L$. Регулярность поля $B^{\alpha}$ получаем из регулярности $B^{-\alpha}$ с помощью очевидных операторныг преобразований. При этом $\left(\partial_{t} B^{\alpha}\right) B^{-\alpha}+B^{\alpha} \partial_{t} B^{-\alpha}=0$, стало быть, $\left\|\left(\partial_{t} B^{\alpha}\right) B^{-\alpha}\right\|=\left\|B^{\alpha} \partial_{t} B^{-\alpha}\right\| \leqslant N$ и лемма доказана.

\section{Список литературы}

1. Романов А.В. Конечномерная предельная динамика диссипативных параболических уравнений // Матем. сб. 2000. Т. 191. №3. С. 99-112.

2. Хенри Д. Геометрическая теория полулинейных параболических уравнений. М.: Мир, 1985.

3. Hopf E. A mathematical example displaing features of turbulence // Comm. Pure Appl. Math. 1948. V. 1. № 4. P. 303-322.

4. Foias C., Prodi G. Sur le comportement global des solutions non-stationnaires des equations de Navier-Stokes en dimension 2 // Rend. Semin. Mat. Univ. Padova. 1967. V. 39. P. 1-34.

5. Ладыженская O. А. О динамической системе, порождаемой уравнениями Навье-Стокса // Записки научн. семин. ЛОМИ. 1972. Т. 27. С. 91-115.

6. Mallet-Paret J. Negatively invariant sets of compact maps and an extension of a theorem of Cartwright // J. Differ. Equat. 1976. V. 22. № 2. P. 331-348.

7. Mane R. On the dimension of the compact invariant sets of certain non-linear maps // Lecture Notes in Math. V. 898. N. Y.: Springer-Verlag, 1981. P. 230-242. 
8. Ладыженская $О$. А. О нахождении минимальных глобальных аттракторов для уравнений Навье-Стокса и других уравнений с частными производными // УМН. 1987. Т. 42. №6. C. $25-60$.

9. Бабин А. В., Вишик М. И. Аттракторы эволюционных уравнений. М.: Наука, 1989.

10. Temam $R$. Infinite-dimensional dynamical systems in mechanics and physics. 2-nd ed. N.Y.: Springer-Verlag, 1997.

11. Чуешов И. Д. Теория функционалов, однозначно определяющих асимптотическую динамику бесконечномерных диссипативных систем // УМН. 1998. Т. 53. № 4. С. 77-125.

12. Mane $R$. Reduction semilinear parabolic equations to finite dimensional $C^{1}$ flows // Lecture Notes in Math. V. 597. N. Y.: Springer-Verlag, 1977. P. 361-378.

13. Романов А. В. Точные оценки размерности инерциальных многообразий для нелинейных параболических уравнений // Изв. РАН. Сер. матем. 1993. Т. 57. № 4. С. 36-54.

14. Романов A.B. Три контрпримера в теории инерциальных многообразий // Матем. заметки. 2000. Т. 68. № 3. С. 439-447.

15. Камаев Д. А. Семейства устойчивых многообразий одномерных параболических уравнений // Матем. заметки. 1996. Т. 60. №1. С. 11-23.

16. Камаев Д. А. Семейства устойчивых многообразий инвариантных множеств систем параболических уравнений // УМН. 1992. Т. 47. № 5. С. 179-180.

17. Brunovsky P., Terescak I. Regularity of invariant manifolds // J. Dyn. Differ. Equat. 1991. V. 3. № 3. P. 313-337.

18. Борисович Ю.Г., Близняков Н.М., Израилевич Я.А., Фоменко Т.Н. Введение в топологию. 2-е изд. (доп.). М.: Наука, 1995.

19. Данфорд Н., Швари, Джс. Т. Линейные операторы. Т. 3. Спектральные операторы. М.: Мир, 1974.

20. Романов А. В. Конечномерность динамики на аттракторе для полулинейных параболических уравнений // Международная конференция, посвященная 90-летию со дня рождения Л.С. Понтрягина. Тезисы докладов. Оптимальное управление и добавления. М.: Изд-во МГУ, 1998. С. 324-325.

21. Yorke J. A. Periods of periodic solutions and the Lipschitz constant // Proc. Amer. Math. Soc. 1969. V. 22. № 2. P. 509-512.

22. Трибель $X$. Теория функциональных пространств. М.: Мир, 1986.

23. Трибель $X$. Теория интерполяции, функциональные пространства, дифференциальные операторы. М.: Мир, 1980.

24. Функциональный анализ. Справочная математическая библиотека / Ред. С.Г. Крейн. М.: Наука, 1973.

25. Левитан Б. М., Сарәсян И. С. Введение в спектральную теорию. М.: Наука, 1970.

26. Ладыженская О. А., Солонников В. А., Уральщева Н. Н. Линейные и квазилинейные уравнения параболического типа. М.: Наука, 1967.

27. Amann H. Global existence for semilinear parabolic systems // J. Reine Angew. Math. 1985. V. 360. P. 47-83.

28. Hoshino H., Yamada Y. Solvability and smoothing effect for semilinear parabolic equations // Funkc. Ekv. 1991. V. 34. № 3. P. 475-494.

29. Хилле Э., Филлипс Р. Функциональный анализ и полугруппы. М.: ИЛ, 1962. 